\title{
Petrochemical evolution of subvolcanic granitoid intrusions within the Late Devonian Mount Pleasant Caldera, southwestern New Brunswick, Canada: comparison of Au versus Sn-W-Mo-polymetallic mineralization systems
}

\author{
Xue-Ming Yang ${ }^{1}$, David R. Lentz ${ }^{1}$, Steve R. McCutcheon ${ }^{2}$ \\ 1. Department of Geology, University of New Brunswick, P.O. Box 4400, Fredericton, \\ New Brunswick, Canada E3B 5A3 (e-mail:m0qm4@unb.ca) \\ 2. New Brunswick Department of Natural Resources, Geological Survey Branch, P. O. Box 50, Bathurst, \\ New Brunswick, Canada E2A 3Z1 (e-mail:steve.mccutcheon@gnb.ca) \\ Date received:September 2, 2003 I Date accepted:January 20, 2004
}

\begin{abstract}
Two spatially and temporally related subvolcanic intrusive suites, the McDougall Brook Granitic Suite (MBG) and the Mount Pleasant Granitic Suite (MPG), occur within the Late Devonian Mount Pleasant Caldera, southwestern New Brunswick. Auriferous greisenized quartz breccias and veins are associated with the MBG, whereas Sn-W-Mo-Bipolymetallic ( $\mathrm{Cu}-\mathrm{Zn}$-Pb-In) mineralization is genetically related to the slightly younger MPG. The low-silica $(<70 \mathrm{wt}$. $\%) \mathrm{MBG}$ and the high-silica (> 74 wt. \%) MPG are calc-alkaline and metaluminous to weakly peraluminous (A/CNK = 0.91 - 1.28), and exhibit some crustal A-type granite affinities. However, compared to the MPG, the MBG has lower amounts of incompatible trace elements, a more pronounced $\mathrm{Nb}$ anomaly, enriched REE patterns with smaller negative Eu anomalies, and lower negative $\mathrm{Ti}, \mathrm{Sr}$, and $\mathrm{Ba}$ anomalies. Trace-element modeling, major-element chemistry, and petrologic evidence indicate that the MBG and MPG may have formed through pulse injection of magmas produced by fractional crystallization from felsic magma chambers at depth, involving an assemblage of clinopyroxene, amphibole, plagioclase, K-feldspar, \pm biotite, \pm zircon, \pm apatite. Supracrustal contamination is evident, resulting in abnormally high alkalies (e.g., K) and high initial $\left({ }^{87} \mathrm{Sr} /{ }^{86} \mathrm{Sr}\right){ }_{\mathrm{i}}$ ratio. The parental magmas may be mainly derived from juvenile materials within the crust. Extremely high $\mathrm{F}(\leq 0.99 \%), \mathrm{Li}(\leq 610 \mathrm{ppm}), \mathrm{Rb}(\leq 1210 \mathrm{ppm}), \mathrm{Cs}(\leq 28 \mathrm{ppm}), \mathrm{U}(\leq$ $43 \mathrm{ppm}), \mathrm{Th}(\leq 50 \mathrm{ppm}), \mathrm{Nb}(\leq 107 \mathrm{ppm}), \mathrm{Ta}(\leq 26 \mathrm{ppm})$, and heavy REE (e.g., $\mathrm{Yb} \leq 36 \mathrm{ppm})$ in the MPG rocks, with very low $\mathrm{K} / \mathrm{Rb}$ ratio $(\leq 56)$ and $\mathrm{Nb} / \mathrm{Ta}$ ratio $(\leq 7.6)$, suggest fluid fractionation played an important role in late-stage magmatic differentiation.

Although gold background contents in both the MBG and MPG are typically low, ranging from $<2$ to $8 \mathrm{ppb}$, W, $\mathrm{Sn}$, and Mo contents in the MBG are much lower than those in the MPG. Gold mineralization appears to be related to the early MBG, as it behaves as a compatible element during magmatic evolution. The MBG is broadly comparable to intrusion-related gold systems elsewhere. However, the MPG has characteristics of evolved topaz-bearing granites commonly associated with Sn-W-Mo-Bi-polymetallic (Cu-Zn-Pb-In) mineralization; the youngest phase of the MPG appears have high potential for generating Sn deposits. Both intrusive suites formed within an epicontinental caldera complex and generally have within-plate geochemical character.
\end{abstract}

\section{RÉSUMÉ}

Deux suites intrusives subvolcaniques spatialement et temporellement apparentées, la suite granitique du ruisseau McDougall (MBG) et la suite granitique du mont Pleasant (MPG), sont présentes à l'intérieur de la caldeira du Dévonien tardif du mont Pleasant, dans le Sud-Ouest du Nouveau-Brunswick. Des filons et des brèches de quartz greisenifiés aurifères sont associées à la MBG, tandis qu'une minéralisation de Sn-W-Mo-Bi-polymétallique (Cu-Zn$\mathrm{Pb}$-In) est génétiquement apparentée à la MPG légèrement plus récente. La MBG à faible teneur en silice ( $<70 \% \mathrm{pds})$ et la MBG à forte teneur en silice (>74\% pds) sont calco-alcalines, métalumineuses à faiblement hyperalumineuses $(\mathrm{A} / \mathrm{CNK}=0,91-1,28)$, et elles affichent quelques affinités granitiques de type A crustales. La MBG présente toutefois, comparativement à la MPG, des quantités inférieures d'éléments traces incompatibles, une anomalie plus prononcée en $\mathrm{Nb}$ et des configurations enrichies de métaux des terres rares avec des anomalies négatives en Eu plus réduites et des anomalies négatives de Ti, $\mathrm{Sr}$ et Ba inférieures. La modélisation des éléments traces, la composition chimique en éléments majeurs et les observations pétrologiques révèlent que la MBG et la MPG pourraient s'être formées par injec- 
tion intermittente de magma produit par cristallisation fractionnée de réservoirs de magma felsique en profondeur et ayant comporté un assemblage de clinopyroxène, d'amphibole, de plagioclase, de feldspath potassique, \pm biotite, \pm zircon et \pm apatite. La contamination supracrustale est évidente; elle a entraîné une présence anormalement élevée d'alcalins (p. ex. K) et un ratio initial élevé $\left({ }^{87} \mathrm{Sr} /{ }^{86} \mathrm{Sr}\right)$. Les magmas parents pourraient principalement provenir de matériaux immatures à l'intérieur de l'écorce. Les teneurs extrêmement élevées de $\mathrm{F}(\leq 0,99 \%)$, de Li $(\leq 610$ parties par million), de $\mathrm{Rb}$ ( $\leq 1210$ parties par million), de $\mathrm{Cs}$ ( $\leq 28$ parties par million), d'U ( $\leq 43$ parties par million, de Th ( $\leq 50$ parties par million), de $\mathrm{Nb}(\leq 107$ parties par million), de $\mathrm{Ta}(\leq 26$ parties par million) et d'éléments de terres rares lourds (p. ex. $\mathrm{Yb} \leq 36$ ) dans les roches de la $\mathrm{MPG}$, avec de très faibles ratios de $\mathrm{K} / \mathrm{Rb}(\leq 56)$ et de $\mathrm{Nb} / \mathrm{Ta}(\leq 7,6)$, permettent de supposer que la cristallisation fractionnée des fluides a joué un rôle déterminant dans la différentiation magmatique du dernier stade.

Même si les teneurs en or de référence, tant dans la MBG que la MPG, sont généralement peu élevées, variant entre moins de deux et huit parties par milliard, les teneurs en W, Sn et Mo de la MBG sont beaucoup plus basses que celles de la MPG. La minéralisation de l'or semble reliée au stade précoce de la MBG, car elle s'est comportée comme un élément compatible pendant l'évolution magmatique. La MBG est d'une façon générale comparable aux systèmes aurifères reliés à des intrusions d'ailleurs. La MPG possède toutefois les caractéristiques des granites topazifères évolués communément associés à une minéralisation de $\mathrm{Sn}$-W-Mo-Bi-polymétallique $(\mathrm{Cu}-\mathrm{Zn}$ - $\mathrm{Pb}$-In); la phase la plus récente de la MPG semble offrir un potentiel élevé de production de gîtes de Sn. Les deux suites intrusives se sont formées à l'intérieur d'un complexe de caldeiras épicontinentales et possèdent généralement un caractère géochimique intraplaque.

[Traduit par la rédaction]

\section{INTRODUCTION}

Intrusion-related gold systems have recently raised great interest among economic geologists, leading to many new discoveries worldwide (McCoy et al. 1997; Thompson et al. 1999; Lang et al. 2000; Lang and Baker 2001; Fan et al. 2003). In southwestern New Brunswick, part of the Canadian Appalachian orogen, several gold deposits and occurrences (e.g., Clarence Stream, Lake George, and Poplar Mountain) have been found to share some similarities with intrusionrelated gold systems elsewhere in the world (McLeod and McCutcheon 2000; Thorne and Lentz 2001; Chi 2002; Lentz et al. 2002; McLeod 2002 ; McLeod and Fyffe 2002; Thorne et al. 2002; Yang et al. 2002a, 2002b; Thorne and Lentz 2003). The model of intrusion-related gold systems has stimulated exploration efforts for gold in the area and has highlighted the need for studies to determine the essential controlling factors for formation of these deposits. Clearly, metal behaviour during magmatic evolution is one of the most important factors controlling mineralization.

A fundamental problem with granitoid intrusions is that the precursor magmas have traveled vertically (or laterally) from their source regions to the upper crustal environment, and hence experienced various geological processes that may have greatly modified their original geochemical signatures; therefore, a comprehensive study involving a multidisciplinary approach is required to deal with the complex problem of their petrogenesis (Clarke 1992). In order to better understand the petrogenesis of a granitoid complex and/or batholith, the temporal and spatial framework of the complex must be established from field relations and geochronological data.

The Late Devonian Mount Pleasant Caldera Complex contains two granitic suites (Kooiman et al. 1986; Sinclair et al. 1988; Sinclair and Kooiman 1990; McCutcheon 1990a, 1990b; McCutcheon et al. 1997, 2001). The older McDougall Brook Porphyritic Microgranite of McCutcheon (1990a) is associated with the early stage of caldera development, whereas the younger Mount Pleasant Porphyry of McCutcheon (1990a) is associated with the final stage of the caldera evolution. These two suites are termed the McDougall Brook Granitic Suite (MBG) and the Mount Pleasant Granitic Suite (MPG) in this study (Fig. 1), because both consist of multiple phases with intrusive contacts. The well-known W-Mo-Bi and Sn-Cu-Zn$\mathrm{Pb}$-In deposits at Mount Pleasant are genetically related to various phases of the MPG (Kooiman et al. 1986; Lentz et al. 1988; Sinclair et al. 1988; McCutcheon 1990b; Sinclair and Kooiman 1990; Inverno 1991; McCutcheon et al. 2001). Interestingly, auriferous greisenized hydrothermal quartz breccias and veins (200 ppb up to $1 \mathrm{~g} / \mathrm{t} \mathrm{Au}$, and up to $27 \mathrm{~g} / \mathrm{t} \mathrm{Ag}$; see Johnson 2003) cut the MBG and its wall-rock, both of which were intruded by the MPG (Figs. 1 and 2).

Previous studies mainly dealt with the Mount Pleasant Granitic Suite (i.e. GRI, GRII, and GRIII) and related mineralization characteristics (see Kooiman et al. 1986; Sinclair et al. 1988; Sinclair and Kooiman 1990; Inverno 1991). In these papers, few complete geochemical data sets on the granites were available. Taylor (1992) reported geochemical data for one sample taken from the MPG, and compared it with the Pleasant Ridge topaz granite to the southwest. Whalen et al. (1996) reported geochemical data for one sample from each of the MPG and MBG suites, and noted geochemical differences and similarities between them. No gold data were reported in these studies. Inverno (1991) concentrated on tin mineralization in the MPG, but did not investigate the MBG. McCutcheon (1990a) and McCutcheon et al. (1997, 2001) focused on the stratigraphy and volcanic sequence correlation within the caldera. He and his coworkers analyzed samples from different phases of the MBG for major elements and selected trace elements as a part of a study on the chemical evolution of magmas within the Mount Pleasant caldera (McCutcheon 1990a; McCutcheon et al. 1997). Again, no gold data were reported in these studies, and no work was done on the petrogenetic linkages between the MPG and MBG, and their relations to gold mineralization. 


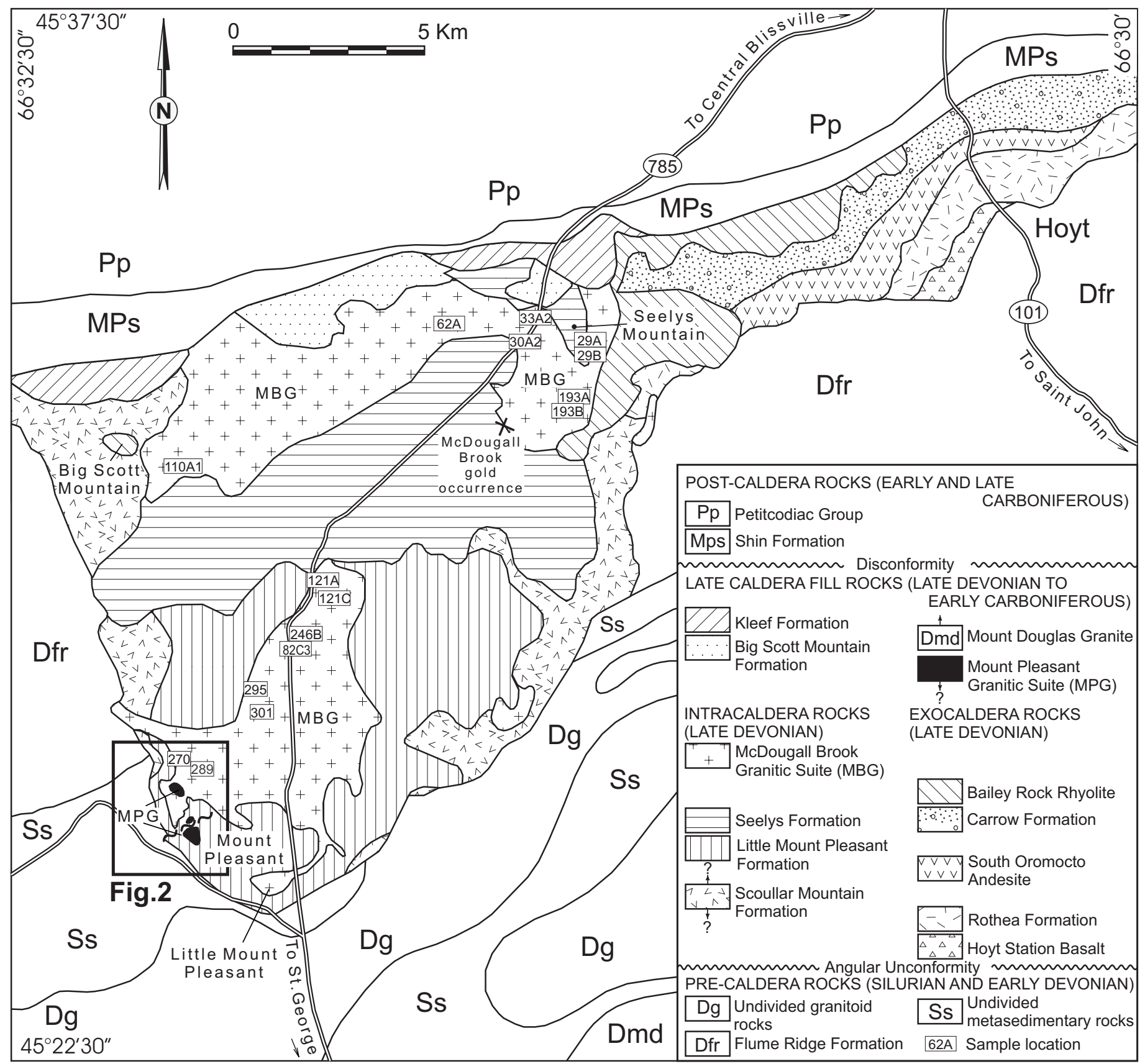

Fig. 1 Schematic geological map of the Mount Pleasant Caldera, southwestern New Brunswick (modified from McCutcheon $e t$ al. 2001). Field relations indicate that the Mount Pleasant Granitic Suite (MPG) cross cuts the McDougall Brook Granitic Suite (MBG). Locations of the McDougall Brook gold occurrence and Figure 2 are also shown.

This study focuses on the petrochemical aspects of these two subvolcanic granitic suites within the Mount Pleasant Caldera in order to determine the essential factors that made one suite produce gold mineralization and the other Sn-W-Mopolymetallic mineralization. On the basis of field relations, petrography, petrochemistry, and trace element modeling, the MBG and MPG are interpreted to be linked through extreme fractional crystallization and supracrustal contamination; fluid fractionation may have played a role in formation of the late MPG. Metal behaviour during the evolution of these magmatic systems indicates that Au behaved as a compatible element, whereas Sn, W, and Mo were incompatible elements. These results can be used to assess the mineralization potential of granitoid intrusions in the area.

\section{GENERAL GEOLOGY}

The Mount Pleasant Caldera (Fig. 1), southwestern New Brunswick, is a northeast-trending elliptical feature with minimum dimensions of 13 by $34 \mathrm{~km}$ (McCutcheon 1990a, 1990b). The caldera is sited on polydeformed Ordovician to Silurian 

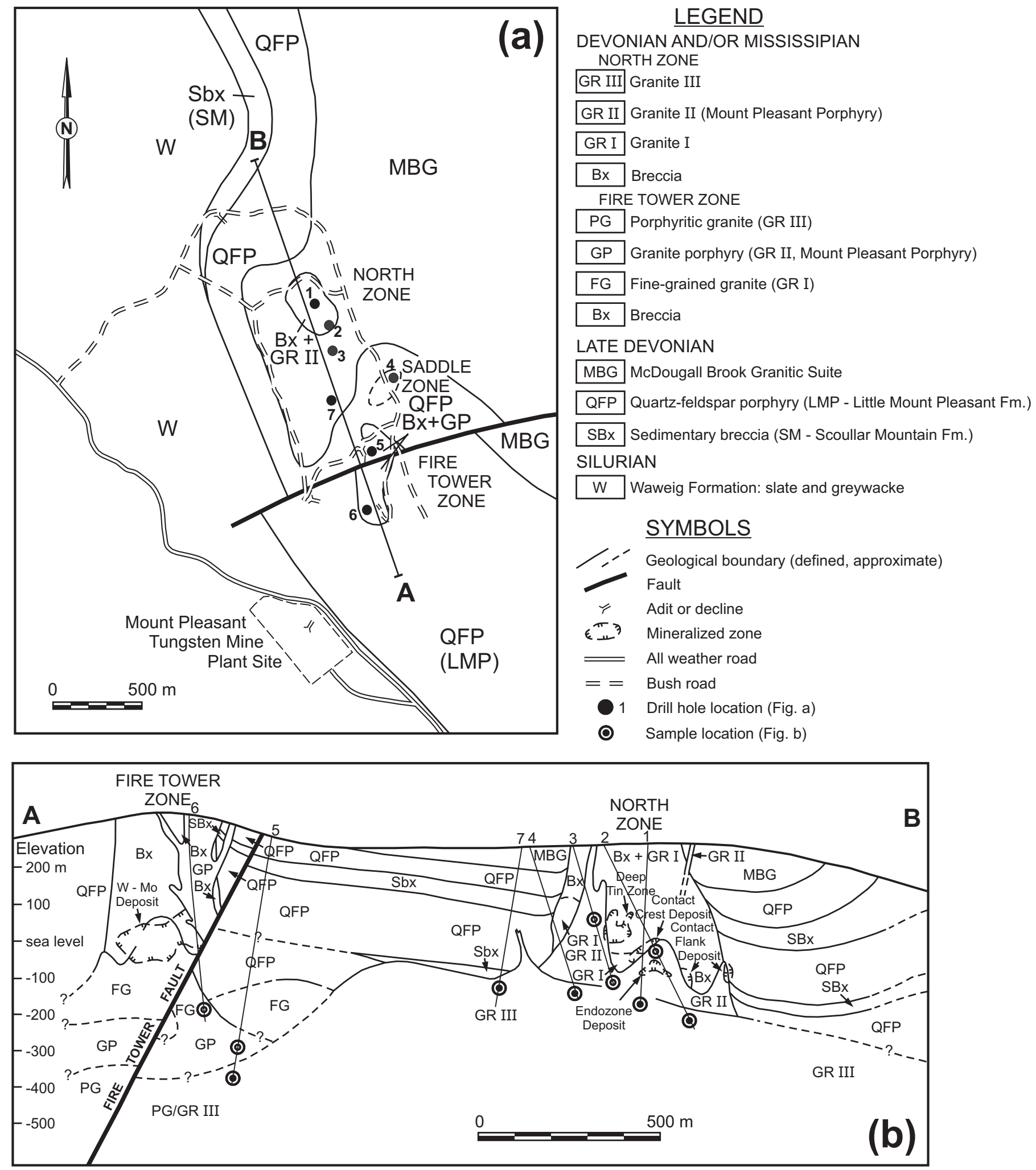

Fig. 2 (a) Simplified geological map of the Mount Pleasant Mine area and (b) cross section A-B through the Fire Tower and North Zones showing the W-Mo-W-Bi and $\mathrm{Sn}$-Cu-Zn-Pb-In deposits and their relations to units ,GRI, GRII, and GRIII, in the order of intrusive sequence, of the Mount Pleasant Granitic Suite (modified from McCutcheon et al. 2001). The least altered samples were taken from drill core. Drill holes shown on this map: 1 - AM96-3; 2 - PRL95-1; 3 - PRL95-2; 4 -LNZ18; 5- NMP89-1; 6 - E8D1; 7 - NMP90-1. 
turbiditic metasedimentary rocks of the Digdeguash and Flume Ridge formations that are thought to form part of the Gander Zone (Fyffe and Fricker 1987). Post-tectonic Middle Mississippian and Pennsylvanian strata overlie the northern part of the caldera, whereas Late Silurian to Devonian granitic rocks of the Saint George Batholith (McLeod et al. 1988; McLeod 1990) form part of its boundary along the southern margin. Rocks within the caldera constitute the Upper Devonian Piskahegan Group that is divided into exocaldera, intracaldera, and late caldera-fill sequences (McCutcheon 1990a, 1990b; McCutcheon et al. 1997, 2001). The Piskahegan Group is largely composed of bimodal volcanic rocks that have been interpreted to have been derived from two parental magmas: mafic with a mantle source and felsic with a lower crustal source (McCutcheon 1990a; McCutcheon et al. 1997). The Mount Pleasant Caldera formed during the transition from a compressional to a transtensional tectonic setting, i.e. during initial development of the Maritimes Basin (McCutcheon et al. 1997). Tectonostratigraphic terrane analysis and geochemical studies (Fyffe and Fricker 1987; Fyffe et al. 1999; Barr and White 1999; Barr et al. 2002) indicate that subduction processes have not taken place in the region since Late Silurian.

Granitic intrusions within the Late Devonian Mount Pleasant Caldera include the McDougall Brook (MBG) and the slightly younger Mount Pleasant (MPG) granitic suites, in the order of intrusive sequence. The MBG is a polyphase suite mainly consisting of marginal feldspar porphyry (MBG1), finegrained (groundmass) porphyritic monzogranite (MBG2), and minor fine-grained equigranular to subporphyritic quartz monzonite(MBG3); these phases occur with intrusive contacts in the sequence of MBG1 $\rightarrow$ MBG2 $\rightarrow$ MBG3 (McCutcheon 1990a; McCutcheon et al. 1997,2001). The northern half of the MBG intrudes intracaldera rocks, including Seelys Formation (rhyolite and ash flow tuffs) to the south, Scoullar Mountain Formation (sedimentary breccia, andesitic and rhyolitic ash flow tuffs) to the west, and exocaldera Bailey Rock Rhyolite to the east. The southern half of the MBG mostly intrudes the Little Mount Pleasant Formation (rhyolitic ash flow tuffs), although small offshoots intrude into the pre-caldera, Early Devonian Flume Ridge Formation and intracaldera Scoullar Mountain Formation to the west (McCutcheon 1990a; Fig. 1). According to McCutcheon (1990a), the MBG represents the transition between a mafic magma that was contaminated by crystal cumulates from overlying felsic magma in a compositionally zoned, high-level subvolcanic magma chamber.

The polyphase MPG (Figs. 1 and 2) is composed of finegrained equigranular granite (GRI), aplitic to porphyritic granite (GRII), and fine- to medium-grained equigranular granite (GRIII), although locally porphyritic and pegmatitc varieties of GRIII are also present (Kooiman et al. 1986; Sinclair et al. 1988; McCutcheon et al. 2001; Figs. 2 and 3). These units have intrusive contacts indicating the intrusive sequence GRI $\rightarrow$ GRII $\rightarrow$ GRIII (Fig. 2b). The GRI unit is heavily brecciated and altered, and is genetically related to early W-Mo-Bi mineralization (Kooiman et al. 1986; Sinclair et al. 1988; McCutcheon et al. 2001). The GRI unit is not included in the
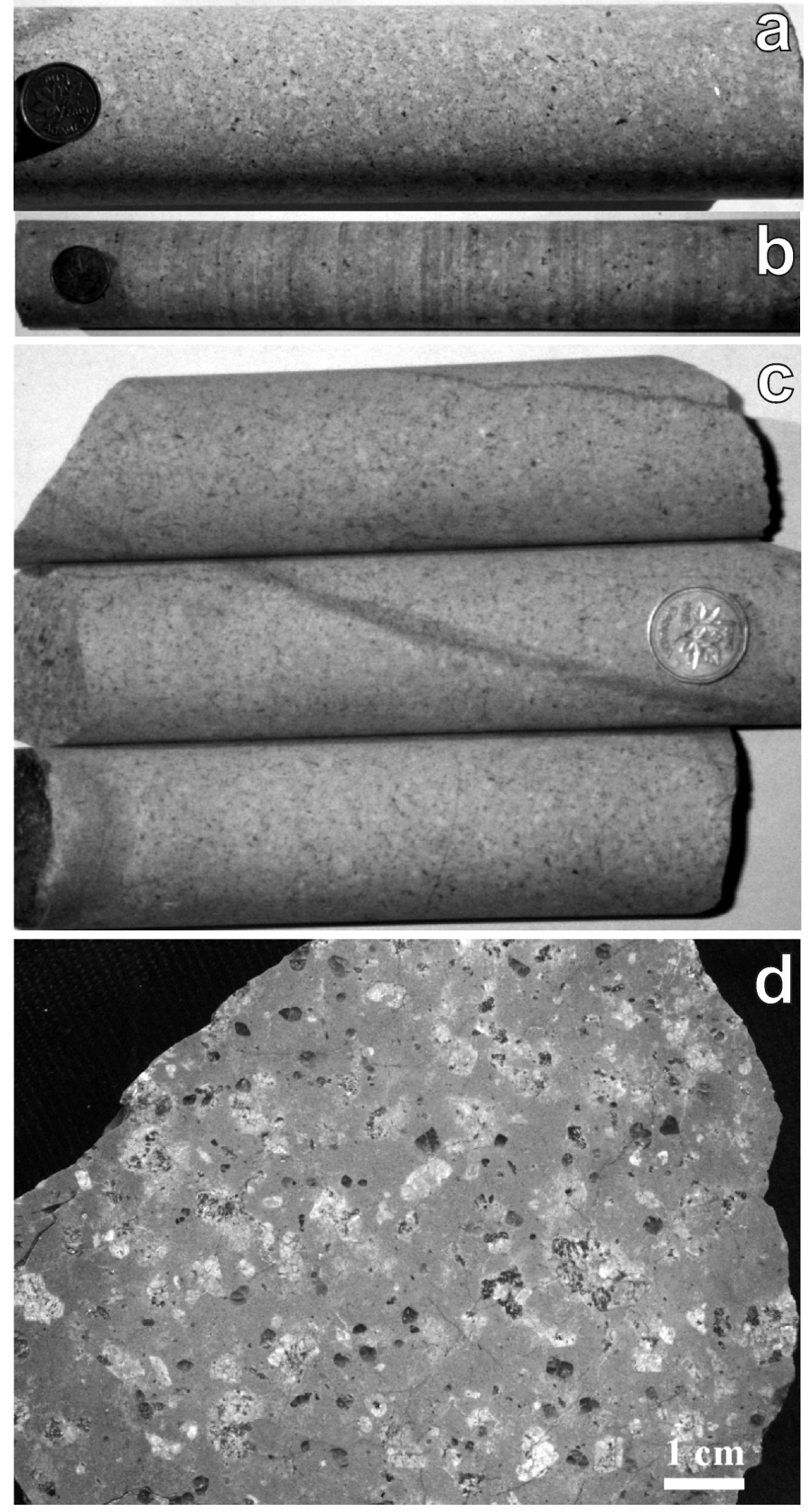

Fig. 3 Photographs of representative drill core samples from the Mount Pleasant Granitic Suite and a typical surface sample from the McDougall Brook Granitic Suite. (a) GRIII: fine-grained, equigranular granite (sample NMP89-11849; (b) GRIII: fine- to medium-grained, equigranular to porphyritic granite (sample LNZ18-1843); (c) GRII: finegrained, equigranular to porphyritic granite (sample NMP-1-1759);(d) porphyritic monzogranite from an outcrop of the McDougall Brook Granitic Suite (sample 33A2, see Fig.1 for location).

present study. The MPG intrudes intracaldera rocks (Scoullar Mountain and Little Mount Pleasant formations) and cuts the MBG (Fig. 2) along the southwestern margin of the caldera (Figs. 1 and 2; Sinclair et al. 1988; McCutcheon et al. 1997, 
2001). McCutcheon (1990a) interpreted the MPG as the top of a zoned, high-level felsic magma chamber that crystallized inward in successive stages.

Radiometric ages are consistent with the field relations mentioned above, indicating that the MPG suite is slightly younger than the MBG suite. $\mathrm{An}{ }^{40} \mathrm{Ar} /{ }^{39} \mathrm{Ar}$ age of ca. $361 \mathrm{Ma}$ was obtained for GRIII (Sinclair and Kooiman 1990) and a U-Pb zircon age of $363.4 \pm 1.8 \mathrm{Ma}$ (Tucker et al. 1998) was obtained for the Bailey Rock rhyolite that is considered to be the extrusive equivalent of the McDougall Brook feldspar porphyry (McCutcheon et al. 1997). Conventional K-Ar data and whole-rock $\mathrm{Rb}-\mathrm{Sr}$ isotopic data yielded less reliable ages of 340-330 Ma for the MPG (Kooiman et al. 1986). A Rb-Sr isochron age of 368 Ma was obtained for the Piskahegan Group (Anderson 1992). These data imply that volcanism and subvolcanic plutonism in the caldera took place during a relatively narrow time interval.

\section{PETROGRAPHY AND RELATED MINERALIZATION}

Based on modal and mesonormative analyses, most of the 27 samples in this study are monzogranite; only three samples from unit MBG1 and one from unit MGB2 are syenogranite (Fig. 4). All of the samples in this study are from subsolvus granite, based on the presence of both plagioclase and Kfeldspar.

\section{McDougall Brook Granitic Suite}

Unit MBG2 mainly consists of porphyritic monzogranite that locally grades to syenogranite. The monzogranite is composed of plagioclase, K-feldspar, quartz, hornblende, and biotite in order of decreasing abundance, with minor pyroxene. The phenocryst assemblage is plagioclase, $\mathrm{K}$-feldspar, and quartz ( $\sim 0 \%$ total abundance), although a few highly altered hornblende (to chlorite) phenocrysts are evident, in which pyroxene cores were noted. Phenocrystic plagioclase forms euhedral to subhedral tabular crystals, ranging from 1 to 12 $\mathrm{mm}$ long, with rectangular cross sections and polysynthetic albite twinning. Glomeroporphyritic plagioclase clusters are common. Plagioclase grains are highly altered, giving them a mottled appearance under both plane and cross-polarized light. The alteration products are mainly saussurite (i.e., finegrained aggregate of epidote group minerals, albite, prehnite, sericite, and possibly clays). K-feldspar (1-12 $\mathrm{mm}$ in grain size) occurs as subhedral, most commonly rectangular, crystals, but rarely euhedral crystals are observed. Coarse patch perthite is common. $\mathrm{K}$-feldspar is also very altered to sericite and clay minerals. Micrographic texture, in which quartz is intergrown in K-feldspar crystals, is present. The quartz forms small, more or less angular grains that are commonly optically continuous over the entire K-feldspar crystal. Phenocrystic quartz generally is less than $4 \mathrm{~mm}$ in size; it commonly occurs as subhedral

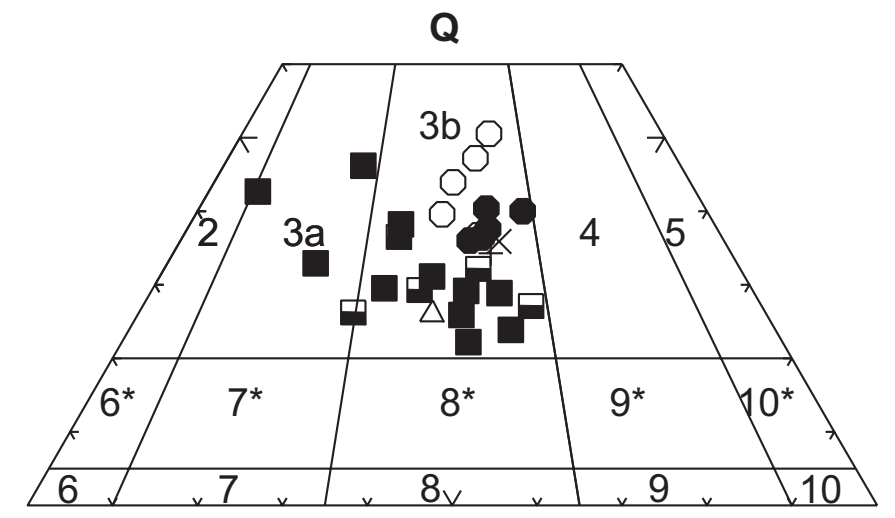

A

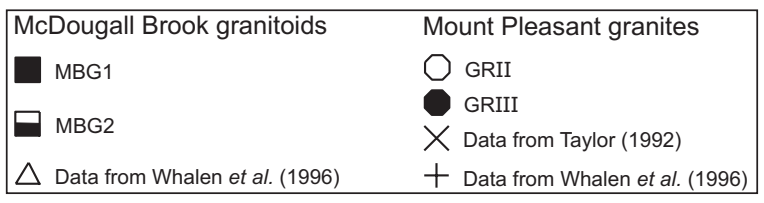

Fig. 4 Mesonormative Q-A-P classification of granitoid samples from the Mount Pleasant Caldera. The fields are after Le Maitre et al. (1989): 2 - alkali feldspar granite, $3 \mathrm{a}$ - syenogranite, $3 \mathrm{~b}$ - monzogranite, 4 - granodiorite, 5 - tonalite. Chemical data are listed in Table 1.

crystals with partly square to hexagonal cross sections, or as anhedral, equant crystals. Groundmass (generally less than 0.5 $\mathrm{mm}$ in grain size) constitutes about $60 \%$ of the rock and has a similar mineralogy to the phenocryst assemblage. Accessory minerals are apatite, titanite, allanite, zircon, and opaque minerals (mainly magnetite).

The less voluminous MBG1 phase occurs on the margin or border of the MBG2 unit, and consists of porphyritic monzogranite (Fig. 3d) and minor equigranular to subporphyritic (containing plagioclase phenocrysts) fine-grained monzogranite. The porphyritic monzogranite contains two populations of subhedral to euhedral feldspar phenocrysts (1-12 mm) with plagioclase generally more abundant than K-feldspar, constituting $30-35 \%$ of the rock. Quartz phenocrysts $(1-4 \mathrm{~mm})$ are minor $(<2 \%)$. The groundmass (generally less than 0.1 $\mathrm{mm}$ ) includes minerals similar to the phenocrysts. Accessory minerals include apatite, zircon, titanite, and magnetite. Most magnetite in the MBG is probably secondary because anhedral magnetite crystals are intimately associated with chlorite that formed by hydrothermal alteration of pyroxene, hornblende, and biotite.

A zone of greisenized quartz breccia and veins about 1500 $\mathrm{m}$ long and several hundred meters wide cuts the MBG and its wall-rocks (Seelys Formation ash flow tuffs). This steeply dipping NW-trending zone (Fig. 1) is notably mineralized and contains significant gold (200 ppb up to $1 \mathrm{~g} / \mathrm{t} \mathrm{Au}$ ) and silver (up to $27 \mathrm{~g} / \mathrm{t} \mathrm{Ag}$ ) (see Johnson 2003). Gold is typically associated with arsenopyrite and pyrite, although the sulphide abundance is low $(<1 \%)$ and unevenly distributed in the quartz breccias and veins. These features are common in intrusion-related gold systems elsewhere (Lang et al. 2000; Lang and Baker 2001). 


\section{Mount Pleasant Granitic Suite}

Units GRII and GRIII of the MPG are similar in mineralogy, and consist mainly of quartz, K-feldspar, plagioclase, and biotite. They may represent two pulses of magma injection from an inward-cooling magma chamber, as indicated by intrusive contacts (Fig. 2b). Unit GRII is mainly composed of porphyritic monzogranite, with local aplitic and seriate textural varieties. Phenocrysts (30-40\%) consist of subhedral to anhedral quartz (0.5 to $1.5 \mathrm{~mm}$ ), anhedral K-feldspar ( 0.5 to $3 \mathrm{~mm}$ ), subhedral to anhedral plagioclase $(0.5$ to $1 \mathrm{~mm})$, and biotite ( 0.5 to $2 \mathrm{~mm}$ ). The groundmass contains minerals similar to the phenocrysts, but the grain size is commonly less than $0.3 \mathrm{~mm}$.

Unit GRIII mainly consists of fine- to medium-grained equigranular monzogranite, although locally porphyritic and pegmatitc varieties are present. Subhedral to anhedral quartz $(30-40 \%)$ is dominant, but euhedral quartz is also observed; grain size ranges from 0.5 to $1.5 \mathrm{~mm}$. K-feldspar usually occurs as anhedral to subhedral crystals from 0.5 to $3.5 \mathrm{~mm}$, which constitute $40-50 \%$ of the rock. Plagioclase (20 to $25 \%$ ) forms subhedral crystals, 1 to $1.5 \mathrm{~mm}$ long. Subhedral biotite flakes, 1 to $3 \mathrm{~mm}$ in size, contain pleochroic halos caused by radiation damage induced by zircon inclusions, form 1-3\%. Opaque minerals include ilmenite, pyrite, and pyrrhotite. Magnetite is associated with chlorite, suggesting that it formed by hydrothermal alteration.

Accessory minerals in both GRII and GRIII are monazite, zircon, ilmenite, columbite, and Li mica. Topaz and fluorite in the GRII and GRIII are both interpreted as products of hydrothermal alteration based on textural relations. However, a few topaz crystals that occur as inclusions in euhedral quartz in GRIII and the presence of primary topaz in an aplitic contact phase of GRII, led Sinclair and Kooiman (1990) to conclude that the MPG can be classified as topaz granite (Manning 1988; Manning and Hill 1990). Magmatic topaz also occurs in leucogranite in the vicinity of Pleasant Ridge and Kedron, as reported by Taylor et al. (1985) and Taylor (1992), who termed the Kedron granite the Bonny River granite. These granite bodies are similar in composition and age to the MPG.

Parts of the aplitic to porphyritic phase of the GRII contain miarolitic cavities, comb quartz layers, and unidirectional solidification textures (UST), suggesting fluid saturation and/ or undercooling of the parental magma (Kirkham and Sinclair 1988). Micrographic intergrowths of quartz and K-feldspar are common in the granite associated with comb quartz layers, both of which reflect a significantly undercooled magma (Fenn 1986; Lentz and Fowler 1992). Detailed drilling and mapping of underground developments indicate that major Sn-Zn$\mathrm{Pb}-\mathrm{Cu}$-In mineralization is closely associated with unit GRII (Kooiman et al. 1986; Sinclair et al. 1988; Sinclair and Kooiman 1990; McCutcheon et al. 2001; Fig. 2b).

In the North Zone at Mount Pleasant, unit GRIII clearly intrudes GRI and GRII (Fig. 2b), which is indicated by sharp contacts that in many places are marked by thin layers of UST (mainly K-feldspar) in the GRIII. Miarolitic cavities (1 $\mathrm{mm}$ to 2 $\mathrm{cm}$ in size) are locally abundant, in which very fine-grained sericite, chlorite, fluorite, and carbonate are present, but arsenopyrite, molybdenite, and other metallic minerals are relatively rare (Sinclair et al. 1988). Despite well preserved fluid saturation textures in GRIII and evidence of hydrothermal activity, only minor tin mineralization is known to be associated with it (Kooiman et al. 1986; Sinclair et al. 1988; Sinclair and Kooiman 1990; McCutcheon et al. 2001). However, further exploration efforts are needed to confirm this conclusion.

Fluid inclusion studies showed that magmatic fluids were predominant in the early stage of mineralization, but more meteoric fluids were involved in late-stage ore-forming processes (Davis and Williams-Jones 1985; Samson 1990). High salinity ( $>30 \mathrm{wt} . \% \mathrm{NaCl}$ equivalent) and high temperature $\left(350-490^{\circ} \mathrm{C}\right)$ fluid is interpreted to have been derived from resurgent boiling, possibly related to magmatic fluids exsolved from the cooling magmas; dilution of this early fluid by convecting meteoric water resulted in low- to moderate-salinity fluids that dominated the inclusion population (Davis and Williams-Jones 1985$)$. Low temperature $\left(180-250^{\circ} \mathrm{C}\right)$ and high-salinity fluid may represent late residual fluid from a change in the pressure regime from dominantly lithostatic to hydrostatic conditions (Davis and Williams-Jones 1985). Clearly, hydrothermal breccias, related pervasive alteration and mineralization (silicification, chloritization, and greisenization) in GRI and GRII (Kooiman et al. 1986) reflect pressure fluctuations of fluids associated with the granitic magmas in the Mount Pleasant deposits. These processes are similar to those that operated in the adjacent True Hill biotite porphyritic granite intrusions (Lentz et al. 1988; Lentz and McAllister 1990; Lentz 1994; Lentz and Gregoire, 1995), suggesting a comparable genesis for all Sn-W-Mo-Bi mineral deposits in the region.

\section{GEOCHEMISTRY}

\section{Analytical procedures}

Ten least-altered granite samples collected from seven drill cores at the Mount Pleasant mine and 17 samples from outcrops of the McDougall Brook Granitic Suite (see Figs. 1 and 2 for sample locations) were analyzed for major elements and selected trace elements, including rare earth elements (REE). The methods used in this study include inductively coupled plasma - optical emission spectrometry (ICP-OES), inductively coupled plasma - mass spectrometry (ICP-MS), instrumental neutron activation analysis (INAA), and X-Ray fluorescence spectrometry (XRF).

The ten samples from the MPG were systematically analyzed with ICP-OES $\left(\mathrm{SiO}_{2}, \mathrm{TiO}_{2}, \mathrm{Al}_{2} \mathrm{O}_{3}, \mathrm{Fe}_{2} \mathrm{O}_{3}\right.$ (total), $\mathrm{MnO}$, $\mathrm{CaO}, \mathrm{MgO}, \mathrm{Na}_{2} \mathrm{O}, \mathrm{K}_{2} \mathrm{O}, \mathrm{P}_{2} \mathrm{O}_{5}, \mathrm{~S}, \mathrm{As}, \mathrm{Ba}, \mathrm{Cd}, \mathrm{Co}, \mathrm{Cr}, \mathrm{Cu}, \mathrm{Ni}$, $\mathrm{Sc}, \mathrm{Sr}, \mathrm{V}$, and $\mathrm{Zn}$ ) and ICP-MS (Rb, Y, Zr, Nb, Cs, La, Ce, Pr, Nd, Sm, Eu, Gd, Tb, Dy, Ho, Er, Tm, Yb, Lu, Hf, Ta, Pb, Th, U, Bi, and Sn) at the GSC-Quebec (Geological Survey of Canada-Quebec), with INAA (Au, Ag, As, Ba, Br, Ca, Co, Cr, 
YANG et al.

Table 1. Analyses of rocks from the Mount Pleasant Granitic Suite, Mount Pleasant Caldera, southwestern New Brunswick

\begin{tabular}{|c|c|c|c|c|c|c|c|c|c|c|c|c|c|}
\hline Sample & 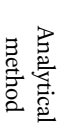 & 讨丞 & 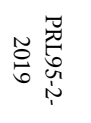 & 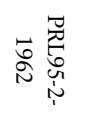 & 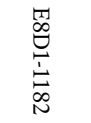 & 党 & $\begin{array}{l}\infty \\
\infty \\
\stackrel{\infty}{N} \\
\text { U. }\end{array}$ & 忑 & 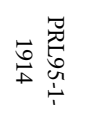 & 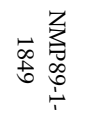 & 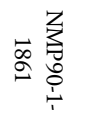 & 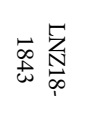 & $\begin{array}{l}3 \\
\vdots \\
\text { c }\end{array}$ \\
\hline Rocks & & GRII & GRII & GRII & GRII & GRII & GRII & GRIII & GRIII & GRIII & GRIII & GRIII & GRIII \\
\hline \multicolumn{14}{|c|}{ Major elements (wt.\%) } \\
\hline $\mathrm{SiO}_{2}$ & 1 & 74.7 & 72.9 & 72.4 & 76.5 & 73.1 & 74.9 & 74.9 & 75.4 & 74.5 & 74.7 & 74.3 & 76.0 \\
\hline $\mathrm{TiO}_{2}$ & 1 & 0.03 & 0.03 & 0.03 & 0.02 & 0.03 & 0.05 & 0.02 & 0.02 & 0.02 & 0.05 & 0.05 & 0.03 \\
\hline $\mathrm{Al}_{2} \mathrm{O}_{3}$ & 1 & 12.60 & 12.69 & 12.06 & 11.77 & 12.33 & 13.10 & 12.15 & 13.28 & 12.60 & 12.49 & 12.11 & 13.12 \\
\hline $\mathrm{Fe}_{2} \mathrm{O}_{3}{ }^{*}$ & 1 & 1.45 & 1.61 & 2.56 & 2.13 & 2.07 & 1.23 & 1.31 & 1.55 & 1.85 & 1.33 & 2.40 & 1.44 \\
\hline $\mathrm{MnO}$ & 1 & 0.04 & 0.18 & 0.17 & 0.09 & 0.05 & 0.06 & 0.08 & 0.10 & 0.06 & 0.07 & 0.06 & 0.07 \\
\hline $\mathrm{MgO}$ & 1 & 0.08 & 0.39 & 0.26 & 0.18 & 0.13 & $<0.01$ & 0.04 & 0.22 & 0.05 & 0.11 & 0.02 & 0.05 \\
\hline $\mathrm{CaO}$ & 1 & 0.79 & 1.88 & 1.47 & 1.20 & 0.78 & 0.43 & 0.36 & 1.08 & 0.74 & 0.79 & 0.63 & 0.36 \\
\hline $\mathrm{Na}_{2} \mathrm{O}$ & 1 & 3.46 & 1.96 & 2.22 & 2.48 & 2.72 & 4.00 & 3.76 & 3.17 & 3.81 & 3.40 & 3.59 & 4.08 \\
\hline $\mathrm{K}_{2} \mathrm{O}$ & 1 & 4.66 & 3.27 & 3.72 & 4.60 & 4.97 & 4.63 & 4.60 & 4.26 & 3.48 & 4.91 & 4.34 & 4.38 \\
\hline $\mathrm{P}_{2} \mathrm{O}_{5}$ & 1 & $<0.025$ & $<0.026$ & $<0.027$ & $<0.026$ & $<0.026$ & $<0.01$ & $<0.028$ & $<0.026$ & $<0.026$ & $<0.027$ & $<0.027$ & 0.00 \\
\hline LOI & & 0.91 & 3.15 & 2.12 & 1.23 & 1.03 & 0.40 & 0.58 & 1.57 & 1.05 & 0.92 & 0.57 & \\
\hline Total & & 98.7 & 98.2 & 97.1 & 100.3 & 97.3 & 98.7 & 97.8 & 100.8 & 98.2 & 98.8 & $\overline{98.4}$ & 99.5 \\
\hline $\mathrm{A} / \mathrm{CNK}$ & & 1.04 & 1.25 & 1.17 & 1.05 & 1.09 & 1.06 & 1.03 & 1.13 & 1.11 & 1.01 & 1.03 & 1.08 \\
\hline$\sigma$ & & 2.1 & 0.9 & 1.2 & 1.5 & 2.0 & 2.3 & 2.2 & 1.7 & 1.7 & 2.2 & 2.0 & 2.2 \\
\hline$\tau$ & & 316 & 352 & 337 & 404 & 324 & 182 & 371 & 449 & 388 & 198 & 183 & 301 \\
\hline \multicolumn{14}{|c|}{ Trace elements (ppm), except for $\mathrm{Au}(\mathrm{ppb})$} \\
\hline S & 2 & 98 & 171 & 284 & 131 & 202 & & 51 & 85 & 52 & 69 & 25 & \\
\hline $\mathrm{Cl}$ & 2 & 241 & 112 & 44 & 57 & 78 & & 258 & 65 & 84 & 75 & 58 & \\
\hline $\mathrm{Rb}$ & 3 & 681.5 & 964.2 & 761.7 & 733.5 & 688.0 & 1054.0 & 932.6 & 813.7 & 1054.3 & 785.4 & 733.0 & 1210.0 \\
\hline Cs & 3 & 7.8 & 27.8 & 11.3 & 5.8 & 5.7 & 27.9 & 20.5 & 10.0 & 17.4 & 12.5 & 5.7 & 21.0 \\
\hline $\mathrm{Ba}$ & 1 & 16.2 & 10.3 & 17.1 & 81.3 & 42.4 & 17.0 & 22.0 & 58.5 & 13.8 & 24.2 & 16.5 & 124.0 \\
\hline $\mathrm{Sr}$ & 1 & 16.5 & 29.5 & 29.9 & 22.9 & 31.5 & 7.0 & 8.3 & 30.9 & 33.1 & 25.8 & 19.9 & $<5$ \\
\hline $\mathrm{Ga}$ & 2 & 33 & 31 & 35 & 30 & 33 & 31 & 33 & 36 & 36 & 29 & 26 & \\
\hline $\mathrm{Ta}$ & 3 & 22.2 & 13.7 & 14.6 & 26.3 & 12.9 & 4.7 & 15.3 & 24.1 & 16.5 & 12.5 & 16.4 & 19.0 \\
\hline $\mathrm{Nb}$ & 3 & 106.5 & 77.7 & 97.2 & 96.8 & 76.9 & 74.0 & 64.5 & 84.4 & 86.3 & 94.6 & 85.5 & 51.0 \\
\hline Hf & 3 & 10.2 & 8.7 & 8.8 & 8.0 & 9.0 & 5.9 & 6.1 & 9.4 & 7.7 & 8.8 & 7.6 & 8.9 \\
\hline $\mathrm{Zr}$ & 3 & 139.4 & 121.9 & 127.5 & 105.4 & 122.9 & 108.0 & 85.0 & 122.1 & 102.9 & 176.4 & 129.2 & 114.0 \\
\hline $\mathrm{Y}$ & 3 & 178.6 & 153.9 & 205.1 & 265.2 & 160.6 & 125.0 & 132.2 & 139.8 & 159.8 & 164.8 & 161.6 & 157.0 \\
\hline Th & 3 & 49.9 & 48.2 & 53.3 & 45.3 & 46.8 & 30.1 & 32.7 & 40.5 & 45.9 & 54.1 & 47.4 & 47.0 \\
\hline $\mathrm{U}$ & 3 & 31.5 & 30.3 & 31.4 & 29.6 & 28.4 & 40.1 & 20.3 & 28.7 & 43.4 & 30.2 & 29.1 & 30.0 \\
\hline $\mathrm{La}$ & 3 & 41.2 & 40.0 & 44.4 & 107.2 & 40.3 & 39.5 & 34.2 & 41.4 & 48.8 & 38.5 & 40.1 & 50.0 \\
\hline $\mathrm{Ce}$ & 3 & 111.7 & 108.6 & 116.0 & 294.7 & 104.9 & 95.7 & 91.1 & 111.2 & 131.1 & 100.5 & 105.8 & 126.0 \\
\hline $\operatorname{Pr}$ & 3 & 13.2 & 12.8 & 13.9 & 33.6 & 12.8 & 12.1 & 10.8 & 13.4 & 15.5 & 11.7 & 12.6 & 17.0 \\
\hline $\mathrm{Nd}$ & 3 & 44.5 & 45.1 & 49.8 & 112.7 & 45.8 & 40.6 & 37.2 & 44.8 & 52.6 & 41.0 & 44.0 & 53.0 \\
\hline $\mathrm{Sm}$ & 3 & 11.9 & 11.6 & 13.4 & 27.0 & 12.2 & 10.4 & 9.7 & 11.5 & 13.0 & 10.9 & 11.2 & 15.0 \\
\hline $\mathrm{Eu}$ & 3 & 0.02 & 0.02 & 0.02 & 0.01 & 0.02 & 0.0 & 0.01 & 0.01 & 0.01 & 0.05 & 0.05 & $<0.05$ \\
\hline $\mathrm{Gd}$ & 3 & 12.1 & 11.0 & 14.2 & 22.9 & 12.6 & 10.0 & 9.5 & 10.9 & 12.3 & 11.3 & 11.4 & 15.0 \\
\hline $\mathrm{Tb}$ & 3 & 2.4 & 2.1 & 2.9 & 4.5 & 2.5 & 2.2 & 1.9 & 2.2 & 2.5 & 2.3 & 2.3 & 3.1 \\
\hline Dy & 3 & 19.0 & 16.4 & 23.0 & 34.4 & 19.4 & 16.2 & 14.8 & 17.6 & 20.2 & 17.9 & 18.5 & 23.0 \\
\hline Ho & 3 & 4.1 & 3.7 & 5.1 & 7.4 & 4.2 & 3.7 & 3.2 & 3.8 & 4.4 & 3.9 & 4.1 & 5.2 \\
\hline $\mathrm{Er}$ & 3 & 14.2 & 13.5 & 17.5 & 25.3 & 14.6 & 13.3 & 11.2 & 13.4 & 15.3 & 14.1 & 14.5 & 18.0 \\
\hline $\mathrm{Tm}$ & 3 & 2.6 & 2.5 & 3.0 & 4.8 & 2.6 & 2.5 & 2.1 & 2.5 & 2.8 & 2.5 & 2.6 & 3.4 \\
\hline $\mathrm{Yb}$ & 3 & 18.8 & 19.1 & 20.9 & 35.7 & 18.9 & 19.2 & 15.2 & 18.9 & 20.6 & 17.9 & 19.0 & 25.0 \\
\hline $\mathrm{Lu}$ & 3 & 2.9 & 3.1 & 3.2 & 5.5 & 2.9 & 3 & 2.4 & 2.9 & 3.1 & 2.8 & 3.0 & 3.7 \\
\hline $\mathrm{Cr}$ & 4 & $<5$ & $<5$ & 11 & $<5$ & $<5$ & 19.6 & $<5$ & $<5$ & $<5$ & $<5$ & 21.6 & \\
\hline $\mathrm{Ni}$ & 2 & 8 & 11 & 8 & 10 & 10 & 7.3 & 16 & 8 & 9 & 6 & 9 & \\
\hline Co & 4 & $<1$ & $<1$ & $<1$ & $<1$ & $<1$ & & $<1$ & $<1$ & $<1$ & $<1$ & $<1$ & \\
\hline $\mathrm{Sc}$ & 1 & 2.76 & 2.71 & 2.82 & 3.04 & 2.96 & & 1.99 & 2.45 & 2.32 & 1.81 & 2.74 & 2.60 \\
\hline $\mathrm{V}$ & 1 & $<10.1$ & $<10.5$ & $<10.7$ & $<10.3$ & $<10.3$ & $<1$ & $<11.2$ & $<10.3$ & $<10.6$ & $<10.7$ & $<10.6$ & \\
\hline $\mathrm{Cu}$ & 1 & 12.2 & 15.7 & 19.4 & 23.1 & 10.9 & $<1$ & $<5.6$ & 24.8 & 55.8 & 10.2 & 326.9 & 3.0 \\
\hline $\mathrm{Pb}$ & 3 & 21.8 & 47.1 & 27.1 & 59.2 & 43.8 & 49.0 & 24.3 & 23.5 & 29.5 & 56.6 & 41.7 & \\
\hline $\mathrm{Zn}$ & 1 & 134.2 & 114.6 & 121.5 & 104.0 & 396.0 & 22.0 & 70.3 & 88.4 & 87.8 & 99.5 & 104.8 & 101.0 \\
\hline $\mathrm{Bi}$ & 3 & 2.8 & 2.3 & 15.5 & 21.7 & 3.1 & & 0.1 & 0.7 & 0.9 & 0.4 & 3.5 & \\
\hline $\mathrm{Sn}$ & 3 & 27.6 & 60.8 & 20.0 & 15.1 & 84.4 & & 43.1 & 18.8 & 145.2 & 31.7 & 115.7 & 0.2 \\
\hline W & 4 & 5 & 24 & 5 & 31 & 13 & & 13 & 11 & 7 & 10 & 7 & 7 \\
\hline Mo & 4 & 8 & $<1$ & 8 & 35 & 37 & & $<1$ & 9 & 14 & 10 & 16 & 10 \\
\hline $\mathrm{Au}$ & 4 & $<2$ & $<2$ & 4 & 7 & $<2$ & & $<2$ & $<2$ & $<2$ & $<2$ & $<2$ & \\
\hline $\mathrm{Ag}$ & 4 & $<5$ & $<5$ & $<5$ & $<5$ & $<5$ & & $<5$ & $<5$ & $<5$ & $<5$ & $<5$ & \\
\hline As & 4 & 2.7 & 9.7 & 12.9 & 42.9 & 8.7 & & 8.7 & 3.5 & 1.0 & 2.5 & 9.1 & \\
\hline Se & 4 & $<3$ & $<3$ & $<3$ & $<3$ & $<3$ & & $<3$ & $<3$ & $<3$ & $<3$ & $<3$ & \\
\hline $\mathrm{Sb}$ & 4 & 0.3 & 0.5 & 0.8 & 0.3 & $<0.1$ & & 0.8 & 0.6 & 0.4 & 0.2 & $<0.1$ & 0.2 \\
\hline
\end{tabular}

Notes: Analytical method: 1 = ICP-OES, 2 = XRF, 3 = ICP-MS, 4 = INAA. $\mathrm{Fe}_{2} \mathrm{O}_{3}$ * total iron oxide; Blank denotes that element was not analyzed. Trace elements are in ppm, except for Au that is in ppb. Symbol "<" indicates element below detection limits.

Sample MP-5 is taken from Taylor (1992), containing 5900 ppm F and 499 ppm Li; Sample SY87-15 is taken from Whalen et al. (1996), containing $6182 \mathrm{ppm} \mathrm{F}$ and 580 ppm Li; Sample SY88-55 is taken from Whalen et al. (1996), containing $863 \mathrm{ppm} \mathrm{F.}$ 
Table 1 (cont'd).

\begin{tabular}{|c|c|c|c|c|c|c|c|c|c|c|c|c|c|c|c|c|c|c|c|}
\hline $\begin{array}{l}\text { Sample } \\
\text { Rocks }\end{array}$ & $\begin{array}{l}\text { Analy. } \\
\text { meth. }\end{array}$ & $\begin{array}{l}\text { 30A2 } \\
\text { MBG1 }\end{array}$ & $\begin{array}{l}\text { 33A2 } \\
\text { MBG1 }\end{array}$ & $\begin{array}{l}\text { 82C3 } \\
\text { MBG1 }\end{array}$ & $\begin{array}{l}\text { 121A } \\
\text { MBG1 }\end{array}$ & $\begin{array}{l}\text { 121C } \\
\text { MBG1 }\end{array}$ & $\begin{array}{l}\text { 193A } \\
\text { MBG1 }\end{array}$ & $\begin{array}{l}\text { 193B } \\
\text { MBG1 }\end{array}$ & $\begin{array}{l}\text { 246B } \\
\text { MBG1 }\end{array}$ & $\begin{array}{r}270 \\
\text { MBG1 }\end{array}$ & $\begin{array}{r}289 \\
\text { MBG1 }\end{array}$ & $\begin{array}{r}301 \\
\text { MBG1 }\end{array}$ & $\begin{array}{l}\text { 29A1 } \\
\text { MBG2 }\end{array}$ & $\begin{array}{l}29 \mathrm{~A} 2 \\
\mathrm{MBG} 2\end{array}$ & $\begin{array}{l}\text { 29B2 } \\
\text { MBG2 }\end{array}$ & $\begin{array}{l}62 \mathrm{~A} \\
\text { MBG2 }\end{array}$ & $\begin{array}{l}\text { 110A1 } \\
\text { MBG2 }\end{array}$ & $\begin{array}{r}295 \\
\text { MBG2 }\end{array}$ & SY88-55 \\
\hline \multicolumn{20}{|c|}{ Major elements (wt.\%) } \\
\hline $\mathrm{SiO} 2$ & 2 & 77.5 & 74.4 & 68.7 & 69.9 & 68.0 & 76.2 & 66.4 & 69.7 & 68.6 & 67.8 & 66.7 & 70.9 & 64.6 & 68.3 & 67.4 & 65.8 & 64.8 & 64.8 \\
\hline $\mathrm{TiO} 2$ & 2 & 0.15 & 0.23 & 0.53 & 0.43 & 0.54 & 0.15 & 0.77 & 0.51 & 0.46 & 0.51 & 0.67 & 0.36 & 0.78 & 0.37 & 0.71 & 0.8 & 0.73 & 0.59 \\
\hline $\mathrm{A} 12 \mathrm{O} 3$ & 2 & 12.0 & 12.9 & 15.9 & 14.0 & 14.7 & 11.6 & 13.8 & 15.5 & 14.7 & 14.6 & 15.6 & 14.4 & 14.1 & 14.2 & 14.0 & 14.3 & 15.7 & 16.2 \\
\hline $\mathrm{Fe}_{2} \mathrm{O}_{3}{ }^{*}$ & 2 & 1.022 & 1.556 & 2.666 & 2.409 & 3.045 & 1.471 & 5.126 & 1.521 & 2.678 & 3.442 & 3.95 & 2.894 & 5.859 & 3.255 & 4.904 & 5.792 & 4.152 & 3.879 \\
\hline $\mathrm{MnO}$ & 2 & 0.02 & 0.02 & 0.02 & 0.07 & 0.06 & 0.01 & 0.14 & 0.01 & 0.06 & 0.19 & 0.11 & 0.05 & 0.11 & 0.1 & 0.11 & 0.09 & 0.13 & 0.15 \\
\hline $\mathrm{MgO}$ & 2 & 0.28 & 0.26 & 0.59 & 0.64 & 0.92 & 0.39 & 0.86 & 0.14 & 0.67 & 0.85 & 0.73 & 0.46 & 0.95 & 0.58 & 0.65 & 0.76 & 0.77 & 1.07 \\
\hline $\mathrm{CaO}$ & 2 & 0.13 & 0.19 & 0.69 & 1.59 & 0.55 & 0.13 & 0.84 & 0.34 & 2.23 & 0.72 & 1.35 & 0.33 & 2.73 & 1.37 & 1.74 & 2.48 & 0.78 & 1.88 \\
\hline $\mathrm{Na}_{2} \mathrm{O}$ & 2 & 1.77 & 2.66 & 3.05 & 3.63 & 3.78 & 0.59 & 1.44 & 3.86 & 3.79 & 2.50 & 4.08 & 2.55 & 3.39 & 2.61 & 2.96 & 3.78 & 2.26 & 2.40 \\
\hline $\mathrm{K}_{2} \mathrm{O}$ & 2 & 6.10 & 5.97 & 5.81 & 4.98 & 5.82 & 8.41 & 7.56 & 5.30 & 5.30 & 6.84 & 5.06 & 5.99 & 4.17 & 6.23 & 4.93 & 4.62 & 7.40 & 6.76 \\
\hline $\mathrm{P}_{2} \mathrm{O}_{5}$ & 2 & 0.02 & 0.03 & 0.13 & 0.11 & 0.15 & 0.03 & 0.24 & 0.11 & 0.12 & 0.13 & 0.19 & 0.08 & 0.26 & 0.09 & 0.21 & 0.24 & 0.22 & 0.17 \\
\hline LOI & & 1.16 & 1.31 & 1.85 & 1.31 & 1.62 & 0.85 & 2.00 & 1.70 & 1.08 & 1.54 & 1.39 & 1.93 & 2.31 & 2.23 & 2.39 & 1.16 & 1.70 & 0.24 \\
\hline Total & & 100.2 & 99.5 & 99.9 & 99.1 & 99.2 & 99.8 & 99.2 & 98.7 & 99.7 & 99.1 & 99.8 & 99.9 & 99.3 & 99.3 & 100.0 & 99.8 & 98.6 & 97.8 \\
\hline ACNK & & 1.23 & 1.15 & 1.27 & 0.98 & 1.09 & 1.13 & 1.14 & 1.22 & 0.92 & 1.14 & 1.07 & 1.28 & 0.94 & 1.05 & 1.05 & 0.91 & 1.19 & 1.10 \\
\hline & & 1.8 & 2.4 & 3.1 & 2.8 & 3.7 & 2.4 & 3.5 & 3.1 & 3.2 & 3.5 & 3.5 & 2.6 & 2.6 & 3.1 & 2.6 & 3.1 & 4.3 & 3.8 \\
\hline & & 68 & 45 & 24 & 24 & 20 & 73 & 16 & 23 & 24 & 24 & 17 & 33 & 14 & 31 & 16 & 13 & 18 & 23 \\
\hline \multirow{2}{*}{\multicolumn{20}{|c|}{ Trace elements (ppm), except for $\mathrm{Au}(\mathrm{ppb})$}} \\
\hline & & & & & & & & & & & & & & & & & & & \\
\hline \\
\hline $\mathrm{Rb}$ & 4 & 276 & 233 & 281 & 198 & 200 & 313 & 334 & 117 & 172 & 323 & 184 & 145 & 100 & 207 & 165 & 164 & 525 & 320 \\
\hline Cs & 4 & 7 & 10 & 17 & 6 & 7 & 12 & 15 & 7 & 5 & 10 & 6 & 8 & 9 & 10 & 9 & 5 & 9 & \\
\hline $\mathrm{Ba}$ & 4 & $<50$ & 340 & 1400 & 1300 & 1600 & 460 & 1100 & 1400 & 1400 & 1950 & 1700 & 900 & 900 & 1200 & 990 & 910 & 1700 & 1663 \\
\hline $\mathrm{Sr}$ & 2 & 70 & 50 & 130 & 190 & 140 & 10 & 50 & 180 & 250 & 160 & 220 & 90 & 200 & 80 & 190 & 210 & 140 & 174 \\
\hline $\mathrm{Ga}$ & & & & & & & & & & & & & & & & & & & 18 \\
\hline $\mathrm{Ta}$ & 4 & $<0.5$ & $<0.5$ & $<0.5$ & $<0.5$ & $<0.5$ & $<0.5$ & $<0.5$ & $<0.5$ & 2.5 & 2.5 & $<0.5$ & $<0.5$ & $<0.5$ & $<0.5$ & 2.6 & $<0.5$ & 2.4 & 0.5 \\
\hline $\mathrm{Nb}$ & 2 & 10 & 30 & 20 & 10 & 10 & 20 & 20 & 40 & 30 & 5 & 5 & 20 & 30 & 5 & 20 & 30 & 20 & 14.3 \\
\hline $\mathrm{Hf}$ & 4 & 7 & 11 & 12 & 10 & 11 & 7 & 12 & 12 & 11 & 12 & 11 & 10 & 12 & 13 & 13 & 14 & 11 & 9.4 \\
\hline $\mathrm{Zr}$ & 2 & 170 & 350 & 480 & 390 & 460 & 220 & 550 & 460 & 480 & 520 & 500 & 480 & 520 & 480 & 540 & 580 & 480 & 387 \\
\hline Y & 2 & 30 & 10 & 60 & 10 & 50 & 30 & 40 & 50 & 30 & 50 & 40 & 30 & 80 & 50 & 70 & 40 & 30 & 25 \\
\hline Th & 4 & 27.6 & 21.9 & 16.9 & 15 & 16.5 & 18 & 14.4 & 14.3 & 14.2 & 14.8 & 15.2 & 12 & 15.7 & 15.9 & 18.8 & 19.6 & 13 & 8.6 \\
\hline U & 4 & 3.9 & 3. & $3 . ?$ & 4.5 & 3.2 & 4.8 & 5.1 & 5.4 & 3.4 & 4.6 & 3.9 & 2.5 & 4.7 & 4 & 4 & 3.9 & 4.3 & 5.4 \\
\hline $\mathrm{La}$ & 4 & 39.4 & 50.8 & 112 & 61.9 & 70.8 & 70.1 & 29.8 & 38.7 & 70.3 & 62.1 & 80.2 & 62.8 & 77.2 & 106 & 80.9 & 71.8 & 73.2 & 47.8 \\
\hline $\mathrm{Ce}$ & 4 & 173 & 162 & 167 & 130 & 156 & 136 & 165 & 120 & 147 & 142 & 169 & 143 & 171 & 178 & 150 & 150 & 168 & 96.9 \\
\hline $\operatorname{Pr}$ & & & & & & & & & & & & & & & & & & & 11.2 \\
\hline $\mathrm{Nd}$ & 4 & 32 & 42 & 60 & 48 & 57 & 64 & 22 & 31 & 62 & 44 & 62 & 54 & 67 & 75 & 60 & 56 & 56 & 42.8 \\
\hline $\mathrm{Sm}$ & 4 & 6 & 7.1 & 11.7 & 9.2 & 10.6 & 10.3 & 6.9 & 7.6 & 10.3 & 9.5 & 11.8 & 9.8 & 13.8 & 13.5 & 12.7 & 12.1 & 12.2 & 7.29 \\
\hline $\mathrm{Eu}$ & 4 & $<0.2$ & 0.9 & 2.6 & 2.3 & 2.5 & 0.7 & 1.7 & 2.1 & 2.6 & 2.6 & 3.2 & 1.7 & 3.4 & 2.9 & 3.4 & 3.3 & 3.3 & 2.17 \\
\hline Gd & & & & & & & & & & & & & & & & & & & 5.95 \\
\hline $\mathrm{Tb}$ & 4 & 0.8 & 0.7 & 1.3 & 1 & $<0.5$ & 0.7 & $<0.5$ & $<0.5$ & 1 & 0.9 & $<0.5$ & 1.3 & 1.4 & $<0.5$ & 1.9 & 1.7 & 1.6 & 0.8 \\
\hline Dy & & & & & & & & & & & & & & & & & & & 4.76 \\
\hline Но & & & & & & & & & & & & & & & & & & & 0.91 \\
\hline Er & & & & & & & & & & & & & & & & & & & 2.64 \\
\hline $\mathrm{Tm}$ & & & & & & & & & & & & & & & & & & & 0.37 \\
\hline $\mathrm{Yb}$ & 4 & 4.9 & 3.4 & 4.8 & 3.6 & 4.1 & 3.9 & 5.8 & 3.8 & 3.9 & 4.2 & 4.6 & 4.3 & 6.4 & 5.2 & 6.4 & 6.2 & 4.8 & 2.42 \\
\hline $\mathrm{Lu}$ & 4 & 0.75 & 0.5 & 0.73 & 0.55 & 0. & 0.59 & 0.86 & 0.56 & 0.6 & 0.65 & 0.7 & 0.65 & 0.96 & 8 & 0.67 & 0.63 & 0.73 & 0.38 \\
\hline $\mathrm{Cr}$ & 4 & $<5$ & $<5$ & $<5$ & $<5$ & $<5$ & $<5$ & 78 & 120 & 5 & $<5$ & $<5$ & $<5$ & $<5$ & $<5$ & $<5$ & $<5$ & 11 & 12.4 \\
\hline $\mathrm{Ni}$ & 2 & 32 & 7 & 15 & 7 & 7 & 3 & 4 & 3 & 5 & 5 & 6 & 5 & 6 & 6 & 11 & 2 & 8 & 6.8 \\
\hline Co & 4 & 2 & 2 & $<1$ & 5 & 5 & $<1$ & 5 & 3 & 2 & 4 & 5 & 4 & 9 & 5 & 6 & 7 & 7 & \\
\hline $\mathrm{Sc}$ & 4 & 5.3 & 6.5 & 8.4 & & 9.4 & 3.7 & 14.9 & & 8.2 & 9.4 & 11 & 9.2 & 18.2 & 11.5 & 15.1 & 16.6 & 12.4 & \\
\hline V & 2 & 10 & 12 & 20 & $<10$ & $<10$ & $<10$ & 30 & 34 & $<10$ & $<10$ & 11 & $<10$ & $<10$ & $<10$ & $<10$ & $<10$ & $<10$ & 37 \\
\hline $\mathrm{Cu}$ & & & & & & & & & & & & & & & & & & & 10 \\
\hline $\mathrm{Pb}$ & & & & & & & & & & & & & & & & & & & 63 \\
\hline $\mathrm{Zn}$ & 4 & $<50$ & $<50$ & $<50$ & 82 & $<50$ & 56 & 113 & $<50$ & $<50$ & 352 & 165 & 121 & 215 & $<50$ & 219 & 202 & 106 & 187 \\
\hline $\mathrm{Bi}$ & & & & & & & & & & & & & & & & & & & \\
\hline Sn & 4 & $<0.02$ & $<0.02$ & $<0.02$ & $<0.01$ & $<0.02$ & $<0.01$ & $<0.02$ & $<0.01$ & $<0.01$ & $<0.01$ & $<0.01$ & $<0.01$ & $<0.02$ & $<0.03$ & $<0.01$ & $<0.01$ & $<0.01$ & \\
\hline W & 4 & $<1$ & $<1$ & $<1$ & $<1$ & $<1$ & 9 & 16 & $<1$ & $<1$ & $<1$ & $<1$ & $<1$ & $<1$ & $<1$ & $<1$ & $<1$ & 5 & \\
\hline Mo & 4 & 5 & 18 & $<1$ & $<1$ & $<1$ & $<1$ & 7 & 4 & 4 & 7 & $<1$ & $<1$ & $<1$ & 3 & $<1$ & $<1$ & $<1$ & \\
\hline $\mathrm{Au}$ & 4 & $<2$ & $<2$ & $<2$ & $<2$ & $<2$ & $<2$ & $<2$ & $<2$ & $<2$ & $<2$ & $<2$ & $<2$ & $<2$ & 6 & 8 & $<2$ & $<2$ & \\
\hline $\mathrm{Ag}$ & 4 & $<5$ & $<5$ & $<5$ & $<5$ & $<5$ & $<5$ & $<5$ & $<5$ & $<5$ & $<5$ & $<5$ & $<5$ & $<5$ & & $<5$ & $<5$ & $<5$ & \\
\hline As & 4 & 39.7 & 20 & 22.1 & 7.4 & 6.8 & 22.7 & 23.1 & 10.1 & 4.4 & 22.3 & 8.2 & 6.7 & 10.5 & 8.3 & 7.5 & 15.5 & 18.8 & \\
\hline $\mathrm{Se}$ & 4 & $<3$ & $<3$ & $<3$ & $<3$ & $<3$ & $<3$ & $<3$ & $<3$ & $<3$ & $<3$ & $<3$ & $<3$ & $<3$ & $<3$ & $<3$ & $<3$ & $<3$ & \\
\hline $\mathrm{Sb}$ & 4 & 1 & 1.9 & 1 & 1.3 & 0.5 & 4.3 & 5.9 & 0.6 & 0.6 & 1.5 & 0.5 & 1.3 & 1.4 & 1.9 & 0.8 & 0.5 & 1.3 & \\
\hline
\end{tabular}



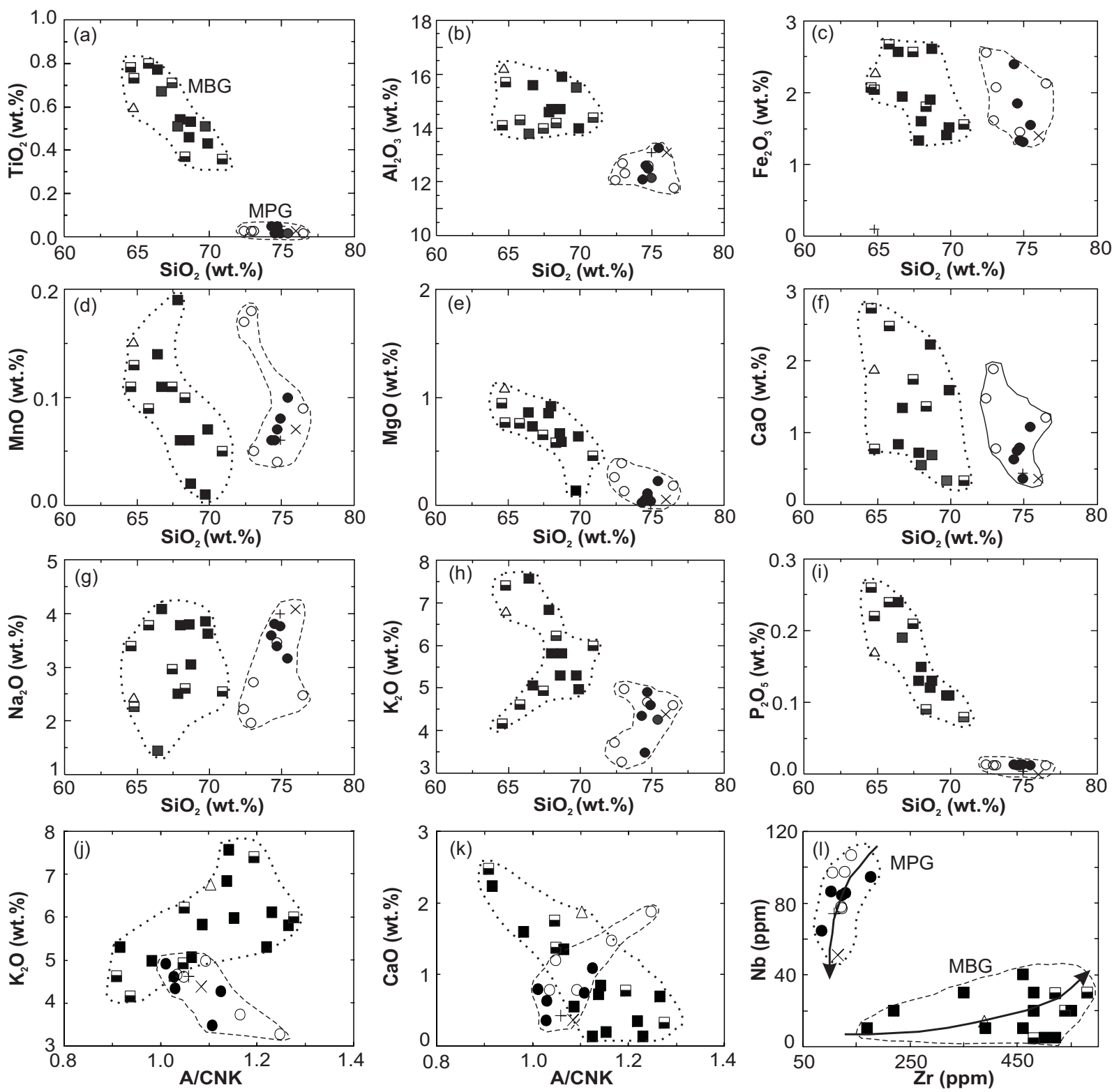

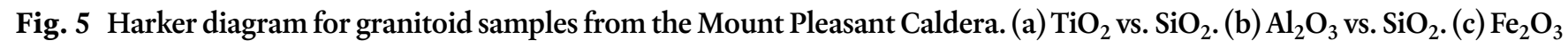
vs. $\mathrm{SiO}_{2}$. (d) $\mathrm{MnO}_{\text {vs. }} \mathrm{SiO}_{2}$. (e) $\mathrm{MgO}$ vs. $\mathrm{SiO}_{2}$. (f) $\mathrm{CaO}$ vs. $\mathrm{SiO}_{2}$. (g) $\mathrm{Na}_{2} \mathrm{O}$ vs. $\mathrm{SiO}_{2}$. (h) $\mathrm{K}_{2} \mathrm{O}$ vs. $\mathrm{SiO}_{2}$. (i) $\mathrm{P}_{2} \mathrm{O}_{5}$ vs. $\mathrm{SiO}_{2}$ (all data in wt.\% in the above plots). ( $\mathrm{j}) \mathrm{A} / \mathrm{CNK}$ vs. $\mathrm{K}_{2} \mathrm{O}$ (wt.\%), where $\mathrm{A} / \mathrm{CNK}=\mathrm{Al}_{2} \mathrm{O}_{3} /\left(\mathrm{CaO}+\mathrm{Na}_{2} \mathrm{O}+\mathrm{K}_{2} \mathrm{O}\right)$, units in moles. $(\mathrm{k}) \mathrm{A} / \mathrm{CNK}$ vs. $\mathrm{CaO}$ (wt.\%). (1) $\mathrm{Zr}(\mathrm{ppm})$ vs. $\mathrm{Nb}$ (ppm); arrows in (1) denote magmatic evolution from early to late stage in the MBG (MBG1 $\rightarrow$ MBG2) and MPG (GRII $\rightarrow$ GRIII) suites. See text for detailed discussion.

Cs, Fe, Hf, Hg, Ir, Mo, Na, Ni, Rb, Sb, Sc, Se, Sn, Sr, Ta, Th, U, W, Zn, $\mathrm{La}, \mathrm{Ce}, \mathrm{Nd}, \mathrm{Sm}, \mathrm{Eu}, \mathrm{Tb}, \mathrm{Yb}$, and $\mathrm{Lu}$ ) at the Activation Labs, Ancaster, Ontario, and with XRF $\left(\mathrm{SiO}_{2}, \mathrm{TiO}_{2}, \mathrm{Al}_{2} \mathrm{O}_{3}\right.$, $\mathrm{Fe}_{2} \mathrm{O}_{3}$ (total), $\mathrm{MnO}, \mathrm{CaO}, \mathrm{MgO}, \mathrm{Na}_{2} \mathrm{O}, \mathrm{K}_{2} \mathrm{O}, \mathrm{P}_{2} \mathrm{O}_{5}, \mathrm{Rb}, \mathrm{Ba}$, $\mathrm{Sr}, \mathrm{Ga}, \mathrm{Nb}, \mathrm{Zr}, \mathrm{Y}$, Th, U, Ce, Cr, Ni, Sc, V, Cu, Pb, Zn, Cl, S, and As) on pressed pellets (Longerich 1995) at the Memorial University of Newfoundland, St. John's. The seventeen samples from the MBG were analyzed with INAA for selected trace elements including REE, also at the Activation Labs; major element data for these samples were taken from the original dataset of McCutcheon (1990a), which were determined by XRF. Certified standard samples SY2 and JG1 (Govindaraju
1994) were used to monitor data quality. Analytical precision of major elements is better than $3 \%$ relative standard deviation (RSD), except for $\mathrm{SiO}_{2}(5 \% \mathrm{RSD})$; and for most trace elements is better than $10 \% \mathrm{RSD}$. Elements for which the analyzed values were beyond $5 \%$ of the recommended values in the standards were not used. The analytical results that satisfied the requirements of precision and accuracy are shown in Table 1 , and are considered to best represent the chemical compositions of the samples. 

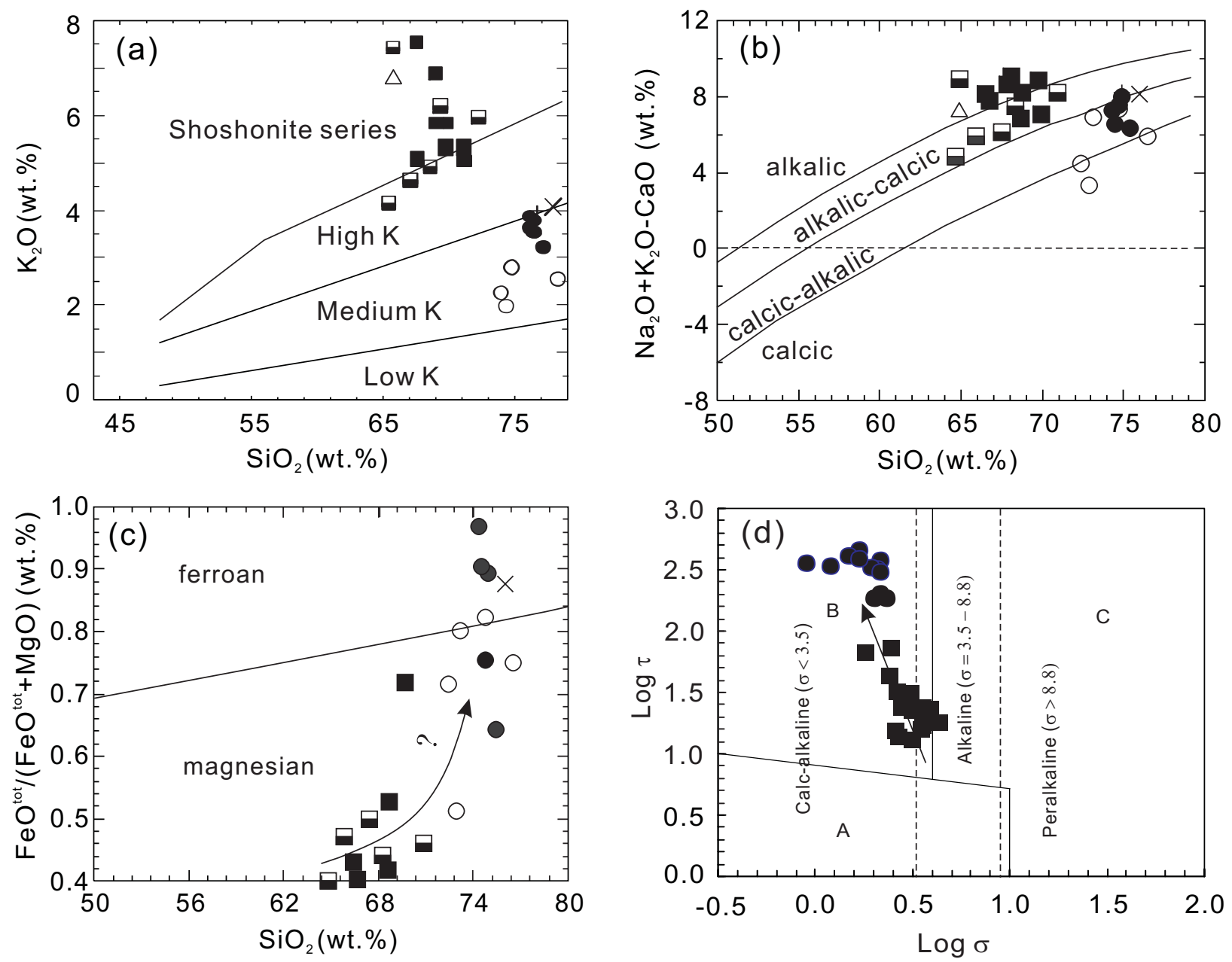

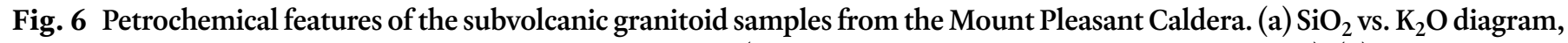
indicating that these granites are characterized by high $\mathrm{K}_{2} \mathrm{O}$ (field boundaries are from Le Maitre et al. 1989). (b) Plot of $\left(\mathrm{Na}_{2} \mathrm{O}+\mathrm{K}_{2} \mathrm{O}-\mathrm{CaO}\right)(\mathrm{wt} . \%)$ vs. $\mathrm{SiO}_{2}$ (wt.\%) (field boundaries for alkalic, alkalic-calcic, calcic-alkalic, and calcic-series granitoids are from Frost et al. (2001)). The variable $\left(\mathrm{Na}_{2} \mathrm{O}+\mathrm{K}_{2} \mathrm{O}-\mathrm{CaO}\right)$ is called MALI (modified alkali-lime index) by Frost et al. (2001), based on the alkali-lime classification of Peacock (1931). (c) $\mathrm{FeO}_{\text {tot }} /\left(\mathrm{FeO}_{\mathrm{tot}}+\mathrm{MgO}\right)(\mathrm{wt} . \%)$ vs. $\mathrm{SiO}_{2}$ (wt.\%) diagram. The arrow indicates the fractionation trend, if the magma is evolving under relatively reduced conditions (i.e. no magnetite is involved in the fractional mineral assemblage). The ferroan and magnesian granite boundary is after Frost et al. (2001). (d) $\sigma-\tau$ diagram for granitoids from the Mount Pleasant Caldera. Solid circles are samples from the Mount Pleasant Granitic Suite; solid squares from the McDougall Brook Granitic Suite. Field A represents lavas of volcanoes situated in anorogenic regions, field B volcanoes in orogenic belts and island arcs, and field $\mathrm{C}$ includes the alkaline derivatives of both, i.e. trachyte, phonolite, tephrite, etc., among which the sodic types are generally linked to A, and potassic types to B. The tectonic discriminant fields are after Rittmann (1973). The calcic- $(\sigma<1.2)$, calc-alkaline- $(1.2-3.5)$, alkaline- $(3.5-8.8)$, and peralkaline-series $(>8.8)$ field boundaries are based on empirical studies of a large database of igneous rocks (Qiu 1986; X. Yang, unpublished data).

\section{Major elements}

Although the MBG has low silica content $\left(\mathrm{SiO}_{2}<70\right.$ wt. \%) relative to the MPG $\left(\mathrm{SiO}_{2}>74 \mathrm{wt}\right.$. \%), both are weakly peraluminous, with four samples from the MBG being metaluminous (Table 1). Aluminum saturation indices $\left(\mathrm{A} / \mathrm{CNK}=\mathrm{Al}_{2} \mathrm{O}_{3} /\right.$ $\left(\mathrm{CaO}+\mathrm{Na}_{2} \mathrm{O}+\mathrm{K}_{2} \mathrm{O}\right)$, molar) range from 0.91 to 1.28 (Table 1). Harker diagrams (Figs. 5a, e and i) show that $\mathrm{TiO}_{2}, \mathrm{MgO}$, and $\mathrm{P}_{2} \mathrm{O}_{5}$ decrease with increasing silica, whereas $\mathrm{Al}_{2} \mathrm{O}_{3}, \mathrm{CaO}, \mathrm{K}_{2} \mathrm{O}$, and $\mathrm{Na}_{2} \mathrm{O}$ show an erratic distribution; $\mathrm{Fe}_{2} \mathrm{O}_{3}$ and $\mathrm{MnO}$ (Figs. $5 \mathrm{c}$ and $\mathrm{d}$ ) are somewhat less erratic. Samples from the MPG are characterized by high $\mathrm{SiO}_{2},\left(\mathrm{Fe}_{2} \mathrm{O}_{3}+\mathrm{FeO}\right) / \mathrm{MgO}$ ratio, and alkalis with $\mathrm{K}_{2} \mathrm{O} / \mathrm{Na}_{2} \mathrm{O}>1$, and low $\mathrm{CaO}, \mathrm{P}_{2} \mathrm{O}_{5}, \mathrm{TiO}_{2}$, and $\mathrm{MgO}$ (Table 1; Figs. $5 \mathrm{c}, \mathrm{e}, \mathrm{g}$, and $\mathrm{h}$ ), thus sharing many similarities with topaz rhyolite, ongonite, and topaz granite (Christiansen et al. 1983, 1984; Christiansen et al. 1986; Kontak 1990; Taylor 1992; Dostal and Chatterjee 1995; Zhu et al. 2001). The MBG and MPG seem to have two distinctive groups, but each group displays a similar variation trend (Figs. 5a-i) except for those on the plots of $\mathrm{A} / \mathrm{CNK}$ versus $\mathrm{K}_{2} \mathrm{O}$ and $\mathrm{A} / \mathrm{CNK}$ versus $\mathrm{CaO}$ (Figs. $5 \mathrm{j}$ and $\mathrm{k}$ ). These trends may indicate that magmatic evolution was controlled by a similar process such as fractional crystal- 
lization, but that minerals involved in the process may have been different in the MBG and MPG.

On the plot of $\mathrm{SiO}_{2}$ versus $\mathrm{K}_{2} \mathrm{O}$ (Le Maitre et al. 1989), samples from the MBG plot in the fields of high- $\mathrm{K}$ and shoshonite series, whereas those from the MPG fall into the field of medium K (Fig. 6a). Using the MALI (modified alkali-lime index) against $\mathrm{SiO}_{2}$ diagram of Frost et al. (2001), the difference in alkalinity between the MBG and MPG is clear: the former belongs to the alkalic-calcic transitional to the alkalic suite, whereas the latter is calcic-alkalic (Fig. 6b). Interestingly, all samples from the MBG are magnesian granites, defining a trend toward higher $\mathrm{FeO}^{\text {total }} /\left(\mathrm{FeO}^{\text {total }}+\mathrm{MgO}\right)$ ratios with increasing silica, but the MPG samples range from magnesian to ferroan granite (Fig. 6c), according to criteria established by Frost et al. (2001).

In terms of the Gottini Index $\tau$ (Gottini 1968) and the Rittmann Serial Index $\sigma$ Rittmann 1957), the lavas of active volcanoes can be divided into two distinctive groups that reflect tectonic setting, i.e. anorogenic and orogenic, as shown in Fig. $6 \mathrm{~d}$. The formulations for the parameters $\tau$ (Gottini 1968) and $\sigma$ (Rittmann 1957) are as follows:

$$
\begin{gathered}
\tau=\left(\mathrm{Al}_{2} \mathrm{O}_{3}-\mathrm{Na}_{2} \mathrm{O}\right) / \mathrm{TiO}_{2} \text { (wt. \%) } \\
\sigma=\left(\mathrm{Na}_{2} \mathrm{O}+\mathrm{K}_{2} \mathrm{O}\right)^{2} /\left(\mathrm{SiO}_{2}-43\right)(\text { wt. \%) }
\end{gathered}
$$

This discrimination diagram was originally proposed by Rittmann (1973), based on plotting of $\log \sigma$ versus $\log \tau$ for 1300 lava samples from active volcanoes worldwide. From mafic through intermediate to felsic igneous rocks, the $\sigma$ value is a very convenient petrochemical indicator, compared to the Peacock index (Peacock 1931), to portray the alkalinity of igneous rocks. Each whole-rock analysis has a defined alkalinity value, if the Rittmann serial index $\sigma$ is appropriately applied (X. Yang, unpublished data).

Most of the samples from the MBG and MPG suites fall into field B, except for six samples from the MBG (three are located in field $\mathrm{C}$, and the other three fall on the boundary between the fields B and C) (Fig. 6d). Samples from the MPG have $\sigma$ values of 1.5 to 2.3 (Table 1), i.e. belonging to the calc-alkaline series, except for one sample (PRL95-2-2019) that has $\sigma$ value of 0.9 , which may be attributed to hydrothermal alteration (i.e. chloritization). Their $\tau$ values are extremely high, ranging from 182 to 449 , consistent with the character of highly differentiated rocks.

Most of the MBG samples are calc-alkaline ( $\sigma$ ranging from 1.8 to 3.3; Table 1$)$, but six samples are weakly alkaline ( $\sigma$ ranging from 3.5 to 4.3 ), consistent with their high alkali contents (Fig. $6 \mathrm{a}$ and b). Their $\tau$ values (13 to 68; Table 1 ) are much lower than those of the MPG samples, indicating that they are less evolved. Notably, an apparent fractionation trend is exhibited on Figure $6 c$, suggesting that the MPG could be linked through differentiation of the MBG.

A few samples (30A2, 33A2, 193A; Table 1) from the MBG1 phase of the MBG show very high $\mathrm{SiO}_{2}$ (up to 77.5 wt. \%) and
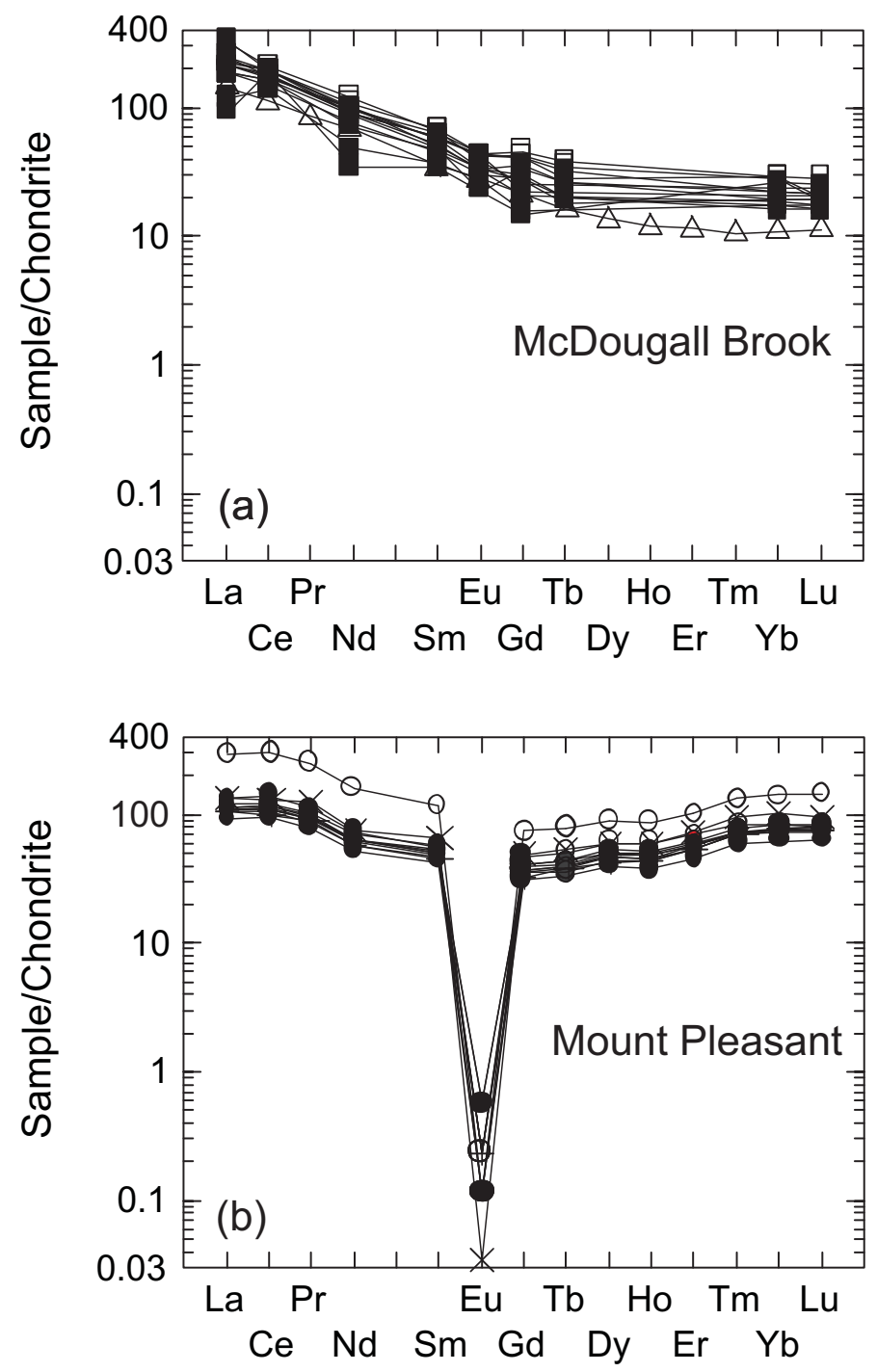

Fig. 7 Chondrite-normalized rare earth element (REE) patterns for granitoids from the Mount Pleasant Caldera. (a) McDougall Brook Granitic Suite samples. (b) Mount Pleasant Granitic Suite samples. Normalizing values for $\mathrm{C} 1$ chondrite are from Sun (1982). Symbols are the same as in Figure 4.

$\mathrm{K}_{2} \mathrm{O}$ (up to 8.41 wt.\%) contents, but relatively low $\mathrm{Na}_{2} \mathrm{O}$ (as low as 0.84 wt.\%) and $\mathrm{Al}_{2} \mathrm{O}_{3}$ (as low as 11.6 wt.\%) contents. These features likely reflect hydrothermal alteration (e.g., silicification, sericitization, and chloritization), as indicated by petrographic observation, and are not the result of magmatic fractionation. Similarly, two samples (PRL95-2-2019 and NMP89-1-1759) from the GRII phase of MPG contain moderately low $\mathrm{SiO}_{2}$ and $\mathrm{Na}_{2} \mathrm{O}$ (Table 1), which also is attributed to hydrothermal alteration (e.g., chloritization). Consequently, interpretations based on alkalis must be considered with caution. As shown in Kontak and Clark (2002), unaltered and altered granitoids are distinguishable on plots of $\mathrm{A} / \mathrm{CNK}$ versus $\mathrm{K}_{2} \mathrm{O}$ and $\mathrm{A} / \mathrm{CNK}$ versus $\mathrm{CaO}$. However, except for these partly altered samples mentioned above, no apparent alteration trends are evident on these plots (Figs. $5 \mathrm{j} \& \mathrm{k}$ ). 

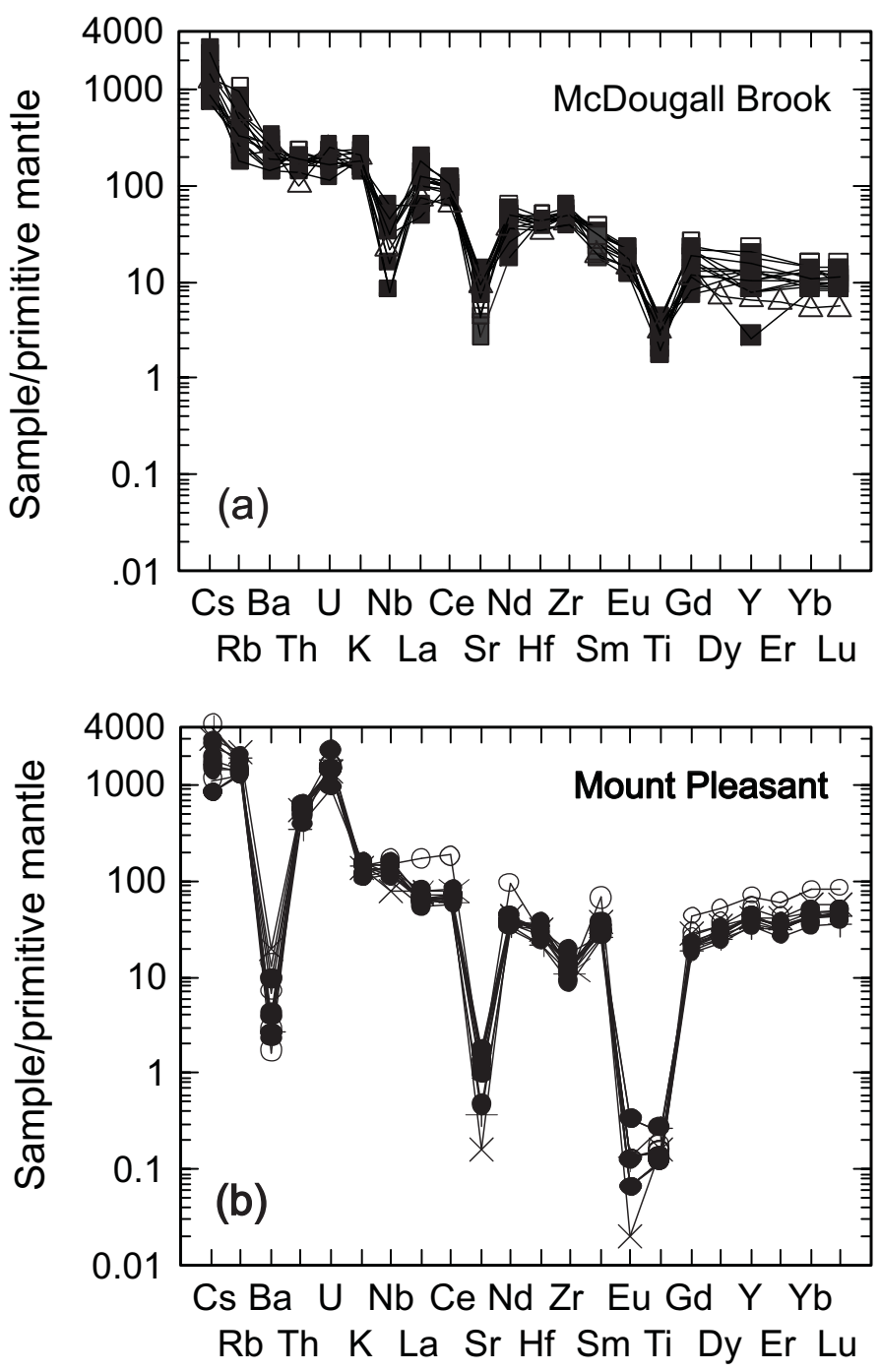

Fig. 8 Primitive mantle-normalized spider diagram for granitoids from the Mount Pleasant Caldera. (a) McDougall Brook Granitic Suite. (b) Mount Pleasant Granitic Suite. Primitive mantle values are from Taylor and McLennan (1985). Symbols are the same as in Figure 4.

\section{Trace and rare earth element (REE) composition}

Trace elements, including REE, in the MBG are distinct from those in the MPG (Table 1). The MBG samples have relatively low incompatible element contents, enriched chondrite-normalized REE patterns $\left[(\mathrm{La} / \mathrm{Yb})_{\mathrm{N}}=3.5-15.7\right]$ with more pronounced negative $\mathrm{Nb}$ anomalies, but small negative Eu anomalies $\left(\mathrm{Eu} / \mathrm{Eu}^{*}=0.43-1.09\right)$, and more pronounced negative $\mathrm{Ti}$, Sr, and $\mathrm{Ba}$ anomalies (Figs. 7 and 8). The flat "birdwing shape" REE patterns (Fig. 7b) of the MPG samples, which are characterized by large negative Eu anomalies $\left(\mathrm{Eu} / \mathrm{Eu}^{*}=\right.$ $0.014-0.002)$ and low $(\mathrm{La} / \mathrm{Yb})_{\mathrm{N}}$ (ranging from 1.4 to 2.0 ) ratios, reflect the highly evolved nature of the MPG. These data are consistent with the major element data (e.g., high $\mathrm{SiO}_{2}$ and alkalis, very low $\mathrm{CaO}$, and high $\tau$ values). From the MBG to MPG, negative Eu anomalies become increasingly

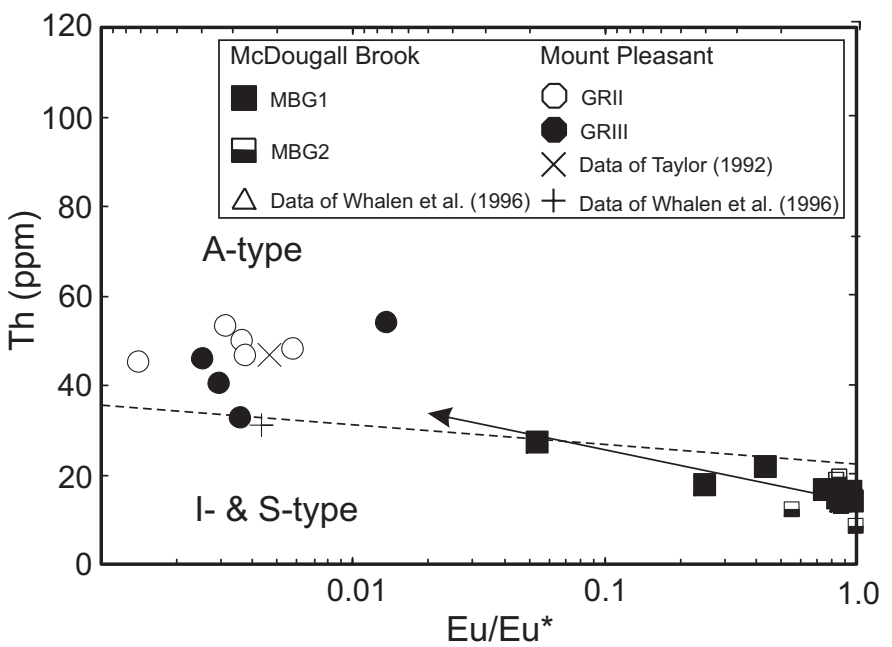

Fig. 9 Variation in $\mathrm{Th}(\mathrm{ppm})$ as a function of the negative $\mathrm{Eu}$ anomaly $\left(\mathrm{Eu} / \mathrm{Eu}^{*}\right)$ for granitoid samples from the Mount Pleasant Caldera. The arrow shows the fractional crystallization trend of a magma crystallizing feldspar. The boundary line between the A- and I- and S-type granite fields is from Eby (1992).

pronounced, with fractionation based on Thenrichment. The MPG samples plot in the A-type granite field, but the MBG samples fall into the I- and S-type field (Fig. 9). If they are associated with fractionation, feldspar must be one of major phases in the crystallizing assemblage (Eby 1992; Christiansen and Keith 1996). It has to be pointed out that highly evolved granites such as the MPG that display some of the geochemical characteristics of A-type intrusions are not necessarily true A-type granites (Whalen et al. 1987; Eby 1990), as no fayalite or sodic hedenbergite that typify "classic" A-type granite occurs in the MPG. Furthermore, petrographic evidence shows that the MPG was saturated with water during crystallization, which also contrasts with the relatively "dry" nature of anorogenic A-type granitoids (Collins et al. 1982; Whalen et al. 1987; Eby 1990).

Relative to I-, S-, A-, and M-type granites (Collins et al. 1982; White and Chappell 1983; Whalen et al. 1987; Eby 1990, 1992), both the MBG and MPG are enriched in $\mathrm{F}(\leq 0.99 \mathrm{wt} . \%), \mathrm{Li}(\leq$ $610 \mathrm{ppm})$ (see Sinclair and Kooiman 1990), Rb ( $\leq 1210 \mathrm{ppm})$, $\mathrm{Cs}(\leq 28 \mathrm{ppm}), \mathrm{U}(43 \mathrm{ppm}), \mathrm{Th}(\leq 50 \mathrm{ppm}), \mathrm{Nb}(\leq 107 \mathrm{ppm}), \mathrm{Zr}$ $(\leq 580 \mathrm{ppm})$, and heavy REE (e.g., $\mathrm{Yb} \leq 36)$ (Table 1$)$, which is typical of crustal A-type felsic magmas (Fig. 10), topaz rhyolite (Christiansen et al. 1983, 1984, 1986), ongonite, and topaz granite (Kontak 1990; Taylor 1992; Zhu et al. 2001). Both the MPG and MBG suites are temporally and spatially related, i.e. part of the Mount Pleasant Caldera, and both intrusive suites formed in a within-plate tectonic setting (McCutcheon et al. 1997), but the petrogenetic relationship between them is less clear. It is noteworthy that $\mathrm{Nb}$ and $\mathrm{Zr}$ behave differently in the MBG and MPG suites. From early to late in time sequence (GRII $\rightarrow$ GRIII), Nb decreases with decreasing Zr in the MPG, 

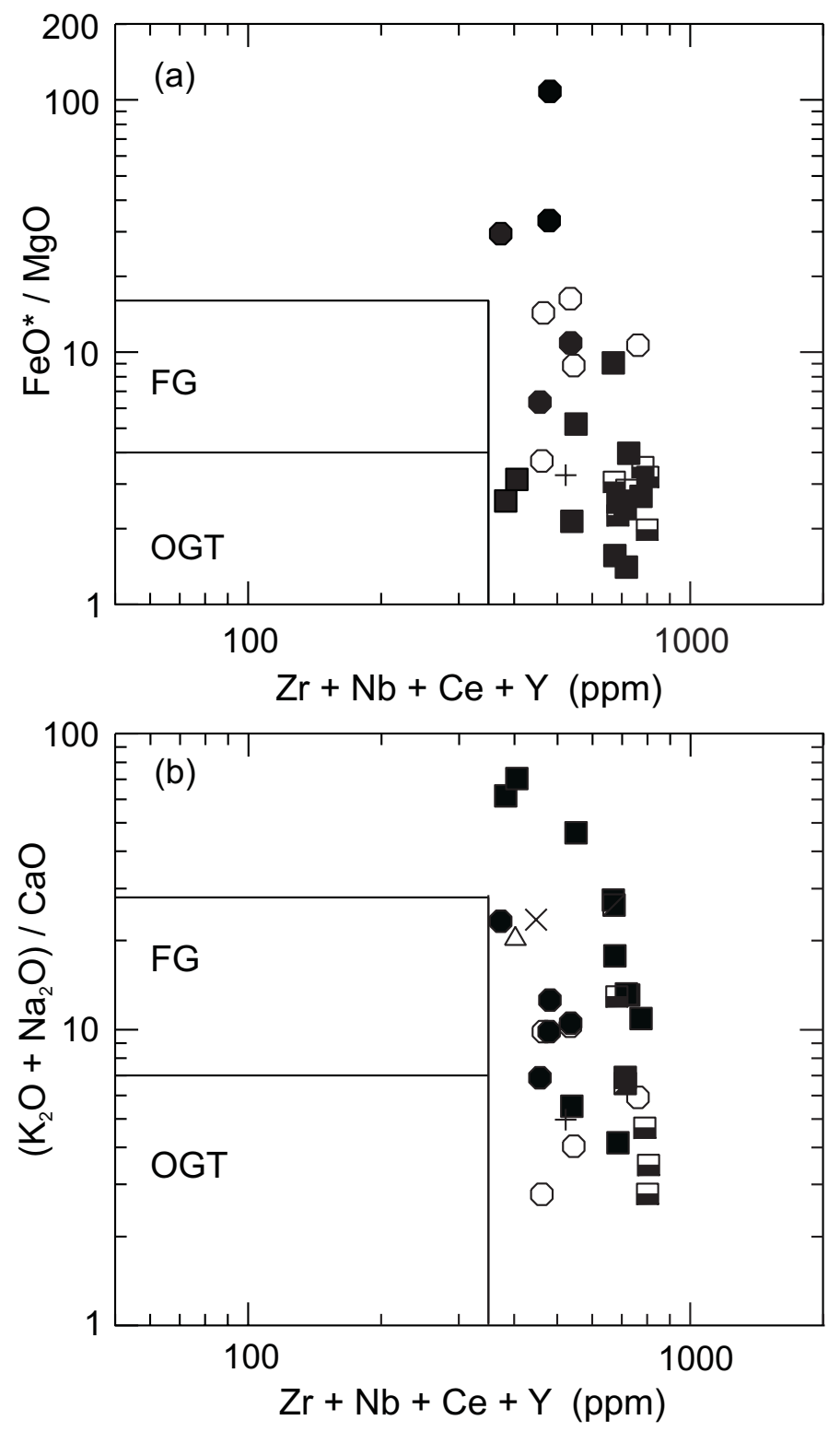

Fig. 10 Geochemical discrimination diagrams showing $(\mathrm{Zr}+\mathrm{Nb}+\mathrm{Ce}+\mathrm{Y})$ plotted against $\mathrm{FeO}^{*} / \mathrm{MgO}(\mathrm{a})$ and $\left(\mathrm{K}_{2} \mathrm{O}\right.$ $\left.+\mathrm{Na}_{2} \mathrm{O}\right) / \mathrm{CaO}(\mathrm{b})$ for granitoid samples from the Mount Pleasant Caldera. A-type granites are located outside of the divided box in each diagram. $\mathrm{FG}=$ fractionated felsic granites and OGT = unfractionated $\mathrm{M}-\mathrm{I}$-, and S-type granites; field boundaries are after Whalen et al. (1987).

whereas $\mathrm{Nb}$ increases with increasing Zr from MBG1 $\rightarrow$ MBG2 in the MBG (Fig. 51).

\section{PETROGENETIC DISCUSSION}

The distribution of major and trace elements in the MBG and MPG seems to indicate two distinctive groups with an apparent "gap" (Figs. 5, 11a-c and 12). It would be easy to ascribe this difference to diverse petrogenetic processes for for- mation of these two granitoid suites within the Mount Pleasant Caldera, without consideration of their field relations, detailed petrographic features, systematic chemical variations, and isotopic compositions. Brophy (1991) noted that compositional gaps, like the ones above, are not uncommon in calc-alkaline magmatic systems. He proposed that fractionation-generated compositional gaps may result from (1) simultaneous interior crystallization and vigorous chamber-wide convection which leads to progressive crystal suspension, (2) cessation of convection when the percentage of suspended crystals reaches the critical crystallinity of the magma, and (3) eventual buoyancy-driven crystal-melt segregation producing a discrete body of fractionated magma that is separated from the initial magma by a compositional gap. Marsh (1996) pointed out that in a magma chamber with phenocrysts, differentiation is controlled by a balance between capture of crystals by the solidification front and crystal settling; massive crystal separation results in less viscous residual magma with discontinuous compositions. Solidification front instability causes silicic melt segregation and produces a separate magma (Marsh 1996).

\section{Isotopic compositions}

Published isotopic data suggest that the MBG and MPG have a similar source, as they have the same $\varepsilon \mathrm{Nd}(\mathrm{t})(-0.2)$ and similar $\delta^{18} \mathrm{O}(7.1$ and $8.2 \sim 8.6 \%$ ) values (see Taylor 1992; Whalen et al. 1996). The slightly lower $\delta^{18} \mathrm{O}$ value of the MBG may be attributed to meteoric water interaction (Whalen et al. 1996). These characteristics suggest that the MBG and MPG either were derived from the same parent magma or from different batches of partial melting of the same protolith. The initial ${ }^{87} \mathrm{Sr} /{ }^{86} \mathrm{Sr}$ ratio for the MPG is 0.7126 (Kooiman et al. 1986), which allows for a crustal source dominated by recycled crustal materials or a mantle source strongly contaminated by supracrustal rocks. The initial ${ }^{87} \mathrm{Sr} /{ }^{86} \mathrm{Sr}$ ratio for the Piskahegan Group is 0.7061 (Anderson 1992), requiring some input from the mantle, consistent with the bimodal nature of the caldera rocks (McCutcheon 1990a; McCutcheon et al. 1997, 2001). A mantle-derived mafic magma could cause partial melting of crustal rocks and generate the felsic magmas responsible for the MBG and MPG. Given low $\mathrm{Sr}$ and high initial ${ }^{87} \mathrm{Sr} /{ }^{86} \mathrm{Sr}$ ratio together with $\varepsilon \mathrm{Nd}(\mathrm{t})$ and $\delta^{18} \mathrm{O}$ values of the MPG rocks, the most likely scenario is that the magma originated from partial melting of juvenile materials and was subsequently contaminated by supracrustal rocks. Based on a systematic study of geochemistry, Taylor (1992) reached the same conclusion for the source material of the Pleasant Ridge topaz granite that has many chemical similarities to the MPG.

\section{Petrogenetic linkage between the MBG and MPG}

Two models are commonly used to explain topaz-bearing granitic rocks: very small degrees of partial melting of continental crustal material that was subjected to an earlier episode of melting (Christiansen et al. 1986; Manning and Hill 1990) and extensive fractionation of felsic magma (Dostal and 

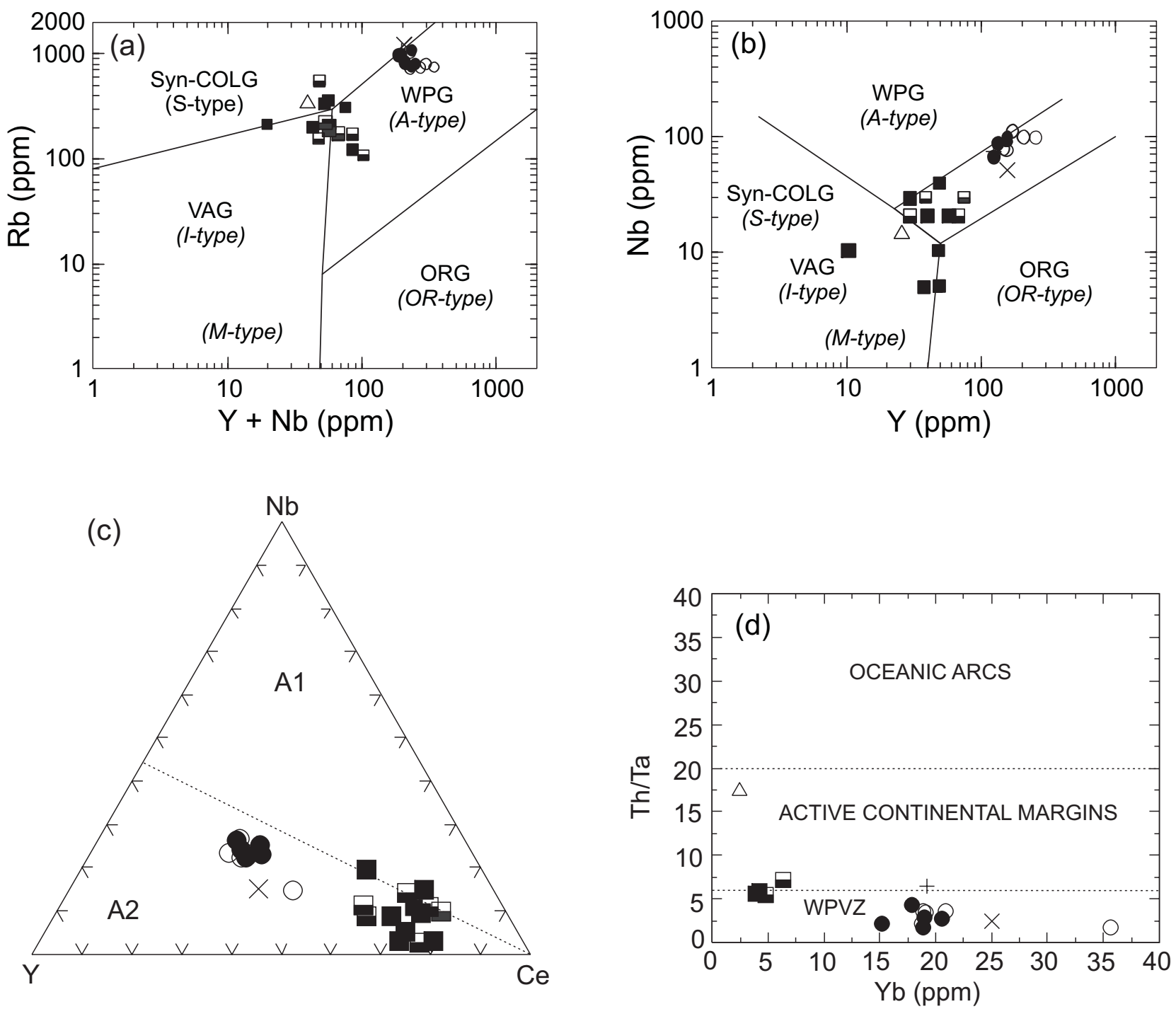

Fig. 11 Tectonomagmatic discrimination diagrams for granitoid samples from the Mount Pleasant Caldera. Log-log plots of $(\mathrm{a})(\mathrm{Y}+\mathrm{Nb}$ ) versus $\mathrm{Rb}$ and $(\mathrm{b}) \mathrm{Y}$ versus $\mathrm{Nb}$. Field boundaries are from Pearce et al. (1984) as modified by Christiansen and Keith (1996). (c) Triangle plot of $\mathrm{Y}-\mathrm{Nb}-\mathrm{Ce}$ (A1 Group granites are characterized by element ratios similar to the mantle, whereas A2 Group granites originated from continental crust or arcs; boundary line between groups is from Eby (1992). (d) Diagram showing $\mathrm{Yb}$ versus Th/Ta; WPVZ = within plate volcanic zones, field boundary is from Gorton and Schandl (2000). Symbols are the same as in Figure 4.

Chatterjee 1995, 2000; Zhu et al. 2001). Generally, the magmas are generated in an extensional tectonic regime (Christiansen et al. 1986; Whalen et al. 1987, 1996). Moreover, extensive liquid fractionation involving thermogravitational diffusion, liquid immiscibility, and vapour-phase transport has also been suggested to explain the petrochemical variations and the substantial enrichment of incompatible trace elements (Christiansen et al. 1983, 1984, 1986; Dostal and Chatterjee 1995, 2000; Zhu et al. 2001).

Large variations in compatible elements, such as $\mathrm{Sr}, \mathrm{Ba}$, and Eu in the MBG and MPG (Table 1, Figs. 7 and 8), rule out the possibility of variable degrees of partial melting of the same source, although small degrees of partial melting can generate high contents of incompatible elements (see Rollinson 1993; Christiansen and Keith 1996). Fractional crystallization, however, not only reduces the abundance of compatible elements, but also increases the concentrations of incompatible elements (e.g., $\mathrm{Li}, \mathrm{Rb}, \mathrm{Cs}, \mathrm{U}, \mathrm{Th}, \mathrm{Nb}$, and $\mathrm{Ta}$ ), without any variations in the isotopic ratios if the effect of supracrustal contamination is not considered.

The petrochemical data presented in this study (Table 1; Figs. 5-9, 11, 12) are modeled assuming that the MBG and MPG were produced by extensive low-T fractional crystallization of the same parental magma, rather than from two batch-melts that evolved separately. The extreme fractional crystallization is interpreted to have taken place mainly at 

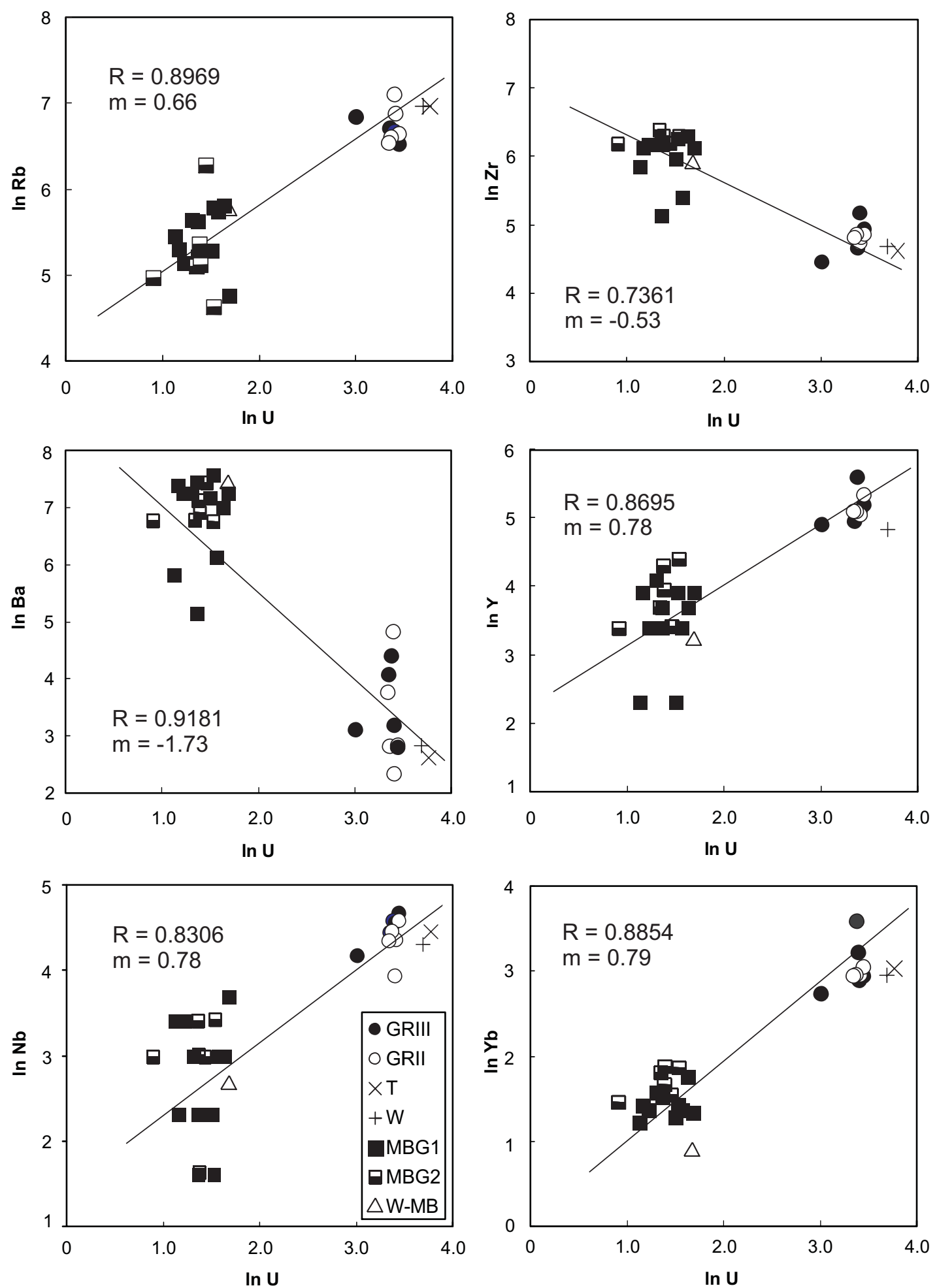

Fig. 12 Representative logarithmic variation diagrams (ppm) for granitoid samples from the Mount Pleasant Caldera. The bulk partition coefficient (D) for each element can be calculated from the equation $D=1-m$, where $m$ is the slope of the line based on least-square regression analysis. $R$ is the correlation coefficient, which is statistically significant at a confidence level of $95 \%$. Symbols are the same as in Figure 4. 


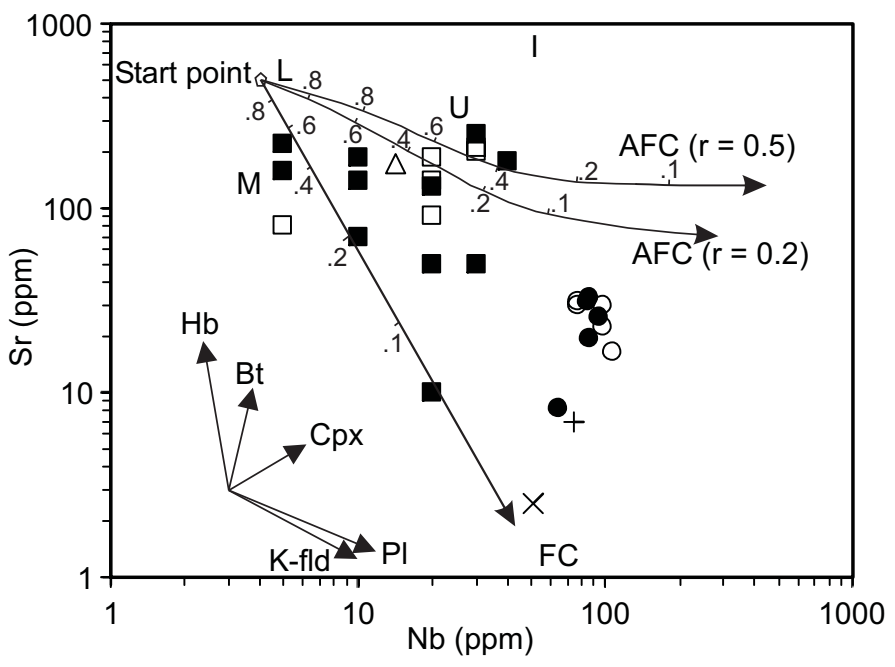

Fig. 13 Log-log plot of $\mathrm{Nb}(\mathrm{ppm})$ versus $\mathrm{Sr}(\mathrm{ppm})$ for granitoid samples from the Mount Pleasant Caldera. Symbols are as those in Fig. 4. Start point is assumed to be: $\mathrm{Nb}=4 \mathrm{ppm}, \mathrm{Sr}=500 \mathrm{ppm}$, equivalent to Late Silurian leucogabbro from the Saint George Batholith (Thorne and Lentz 2002). The upper continental crust (Taylor and McLennan 1985) is used as the contaminant $(\mathrm{Nb}=25$ $\mathrm{ppm}, \mathrm{Sr}=350 \mathrm{ppm})$ in AFC modeling. Bulk coefficients are calculated based on the method of Allégre et al. (1977) for processing the dataset (Table 1): $\mathrm{D}_{\mathrm{Sr}}=1.81, \mathrm{D}_{\mathrm{Nb}}=0.22$; see text for detailed discussion. General trajectories for fractional crystallization (FC) and for assimilation-fractional crystallization (AFC) are shown for comparison, with marks of residual magma fraction. The equations of $\mathrm{FC}$ and $\mathrm{AFC}$ are taken from Rollinson (1993) for the calculations. Ratio of mass assimilated to mass fractionation $(\mathrm{r})$ was simulated for 0.2 and 0.5 , which is shown for the AFC curves. General theoretical vectors produced by Rayleigh fractionation of single phases and their effect on melt composition are also shown for reference: plagioclase (Pl), K-feldspar (K-fld), biotite $(\mathrm{Bt})$, hornblende $(\mathrm{Hb})$, and clinopyroxene $(\mathrm{Cpx})$; mineral-melt partition coefficients are from the compilations of Rollinson (1993). $\mathrm{U}$ - upper continental crust (Taylor and McLennan, 1985) $(\mathrm{Nb}=25 \mathrm{ppm}, \mathrm{Sr}=350 \mathrm{ppm})$; L - lower continental crust (Weaver and Tarney 1984) $(\mathrm{Nb}=5 \mathrm{ppm}, \mathrm{Sr}$ $=569 \mathrm{ppm}$ ); $\mathrm{M}$ - average $\mathrm{N}$-type MORB (Saunders and Tarney 1984) $(\mathrm{Nb}=2.5 \mathrm{ppm}, \mathrm{Sr}=136 \mathrm{ppm}) ; \mathrm{I}$ - average OIB (Sun 1980 $)(\mathrm{Nb}=53 \mathrm{ppm}, \mathrm{Sr}=800 \mathrm{ppm})$.

depth, as indicated by two separate compositional groups, but with the same evolutionary trends for the MBG and MPG (Figs. 5, 6c \& d, 9, 11, and 12). However, supracrustal contamination of selective components is also reflected in some major elements (Fig. 5) and high initial ${ }^{87} \mathrm{Sr} /{ }^{86} \mathrm{Sr}$ ratio, as well as trace elements such as $\mathrm{Rb}$ and $\mathrm{Cs}$ (Fig. 12; Table 1). The erratic distribution of the alkali elements is interpreted as the manifestation of contamination or alteration, whereas the tightly constrained distribution of $\mathrm{TiO}_{2}, \mathrm{P}_{2} \mathrm{O}_{5}$, and $\mathrm{MgO}$ is interpreted as primary features. No alteration trends are apparent on Figures $5 \mathrm{j}, \mathrm{k}$ (cf. Kontak et al. 2002). Figure 13 illustrates the log-log plot of incompatible $(\mathrm{Nb})$ versus compatible (Sr) elements for the samples from the MBG and MPG, in which the samples are mainly confined in the field between fractional crystallization (FC) and assimilation-fractional crystallization (AFC) $(\mathrm{r}=0.5)$ curves. Assuming a leucogabbro (Thorne and Lentz 2002) magma as the starting point and using upper continental crust (Taylor and McLennan 1985) as contaminant, the FC and AFC paths (Fig. 13) can be constructed with the equations of Rollinson (1993), given the bulk partition coefficients $\left(\mathrm{D}_{\mathrm{Sr}}\right.$ and $\mathrm{D}_{\mathrm{Nb}}$ ) estimated by the method of Allégre et al. (1977) (see details below). The parameters used in the modeling are given in the caption of Fig. 13. General vectors produced by Rayleigh fractionation of single phases (e.g., plagioclase, K-feldspar, biotite, hornblende, and clinopyroxene) and their effects on melt composition are also shown for comparison. The compositions of average N-type MORB (Saunders and Tarney 1984), average OIB (Sun 1980), lower continental crust (Weaver and Tarney 1984), and upper continental crust (Taylor and McLennan 1985) are plotted on Fig. 13, which constrains the probable sources for the granitoids from the Mount Pleasant Caldera. All these data suggest that magma evolution was controlled by extreme fractional crystallization plus selective supracrustal contamination. Figure $6 \mathrm{c}$ indicates that fractional crystallization may have proceeded under relatively reduced conditions, as high $f\left(\mathrm{O}_{2}\right)$ would have resulted in crystallization of magnetite and depleted the residual magma in iron.

A simplified calculation was performed to test the probability of petrogenetic connection between the MBG and MPG through fractional crystallization. Preliminary trace-element modeling, together with major-element petrochemical evidence, indicates that the mineral assemblage involved in fractional crystallization was clinopyroxene, amphibole, plagioclase, K-feldspar, biotite, zircon, and apatite (Fig. 13). Some minerals rich in light REE (e.g., monazite and allanite) may also occur as separate phases, although only a very small proportion is required. The most evolved sample (NMP89-1-1849) from the MPG is equivalent to $10-15 \%$ residual melt (for most incompatible elements, such as $\mathrm{Nb}, \mathrm{Ta}$, and $\mathrm{HREE}$ ) generated by the most primitive sample (295) from the MBG that is assumed to represent the composition of the parental magma in terms of a fractional crystallization model (Christiansen and Keith 1996) and the estimated bulk partition coefficient (D) as described below. Inconsistency with a lower degree of fractionation for incompatible elements (e.g., $\mathrm{Rb}$ ) is interpreted to imply that the enrichment of the high field strength elements, such as $\mathrm{Nb}, \mathrm{Ta}$, and HREE, may require fluid involvement. Dostal and Chatterjee $(1995,2000)$ showed that fluid fractionation could have resulted in differential enrichment of these elements to the level observed in ca. 370 Ma topaz leucogranite from South Mountain Batholith in Nova Scotia. Compared to their data, the present study indicates that fluid fractionation may have played an important role in the extreme enrichment of these elements (e.g., Nb, Ta, and HREE) in the MPG (see discussion below). 


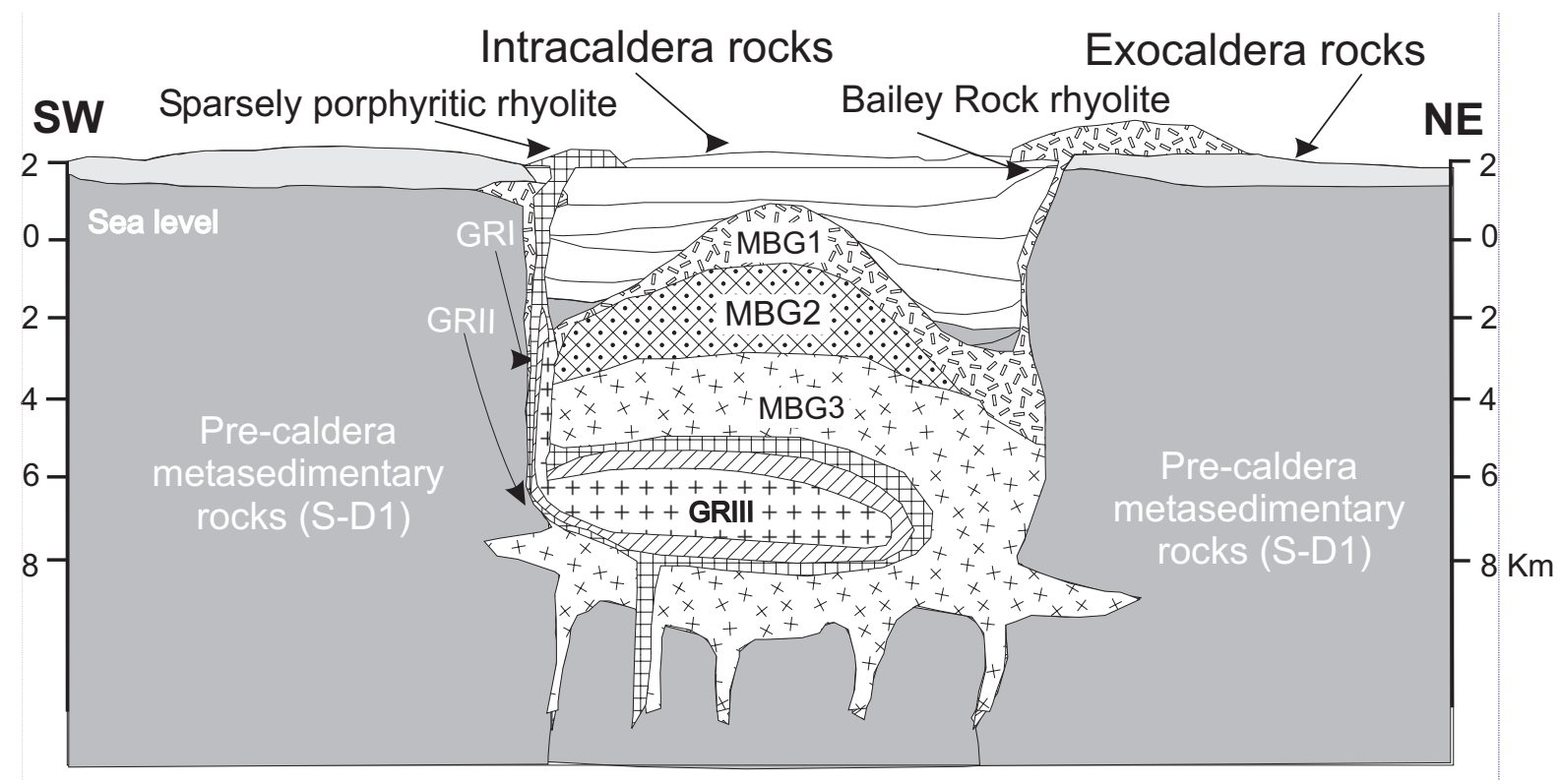

Fig. 14 A hypothetical model for the origin of the granitic suites in the Mount Pleasant Caldera (modified from McCutcheon et al. 1997). Fractional crystallization took place in the magma chamber at depth, cooling and crystallizing inward to the center of the chamber and resulting in zoning in composition and physiochemical variables. The marginal zone of the chamber is more contaminated by supracrustal rocks. Sequential pulses of magma injection were emplaced into the upper crustal rocks (the Piskahegan Group within the caldera). High F, Li and $\mathrm{H}_{2} \mathrm{O}$ contents in the magma enhanced the protracted crystallization, resulting in extreme enrichment in some incompatible elements (e.g., $\mathrm{Rb}, \mathrm{Li}, \mathrm{Cs}, \mathrm{Nb}, \mathrm{Ta}, \mathrm{HREE}, \mathrm{Sn}$, and $\mathrm{W}$ ), and depletion in compatible elements such as $\mathrm{Eu}, \mathrm{Sr}, \mathrm{Ba}, \mathrm{Ti}$, and $\mathrm{P}, \mathrm{Au}$, and $\mathrm{Zr}(\mathrm{Hf})$ in the late MPG.

This model of fractional crystallization coupled with fluid fractionation plus supracrustal contamination proposed in this study (Fig. 14) differs from the previous model by McCutcheon (1990a), wherein he suggested that two different magmas (i.e. two stages of partial melting of lower crust) evolved separately in high-level chambers by AFC processes to give rise to the different phases. McLeod (1990) proposed that highly evolved granites like the MPG may be derived from partial melting of a F-rich source (crust) at depth, which subsequently fractionated repeatedly in a higher level subvolcanic chamber. In that model, the origin of the MBG and its relation to the Mount Pleasant granites were not discussed.

\section{Metal behaviour during fractional crystallization}

In terms of the Rayleigh fractionation law, the partition coefficient D of a hygromagmatophile element such as $\mathrm{U}$ can be assumed to be zero in order to evaluate geochemical behaviour of the other elements in a fractionating magmatic system. An equation for this relation is deduced based on this assumption (Allégre et al. 1977):

$$
\ln \mathrm{C}_{\mathrm{L}}=\ln \mathrm{C}_{\mathrm{o}}+(1-\mathrm{D}) \ln \left(\mathrm{C}^{*}{ }_{\mathrm{L}} / \mathrm{C}^{*}{ }_{\mathrm{o}}\right)
$$

where $\mathrm{C}_{\mathrm{L}}$ and $\mathrm{C}_{\mathrm{o}}$ denote the concentration of an element of interest in residual melt and parental melt, respectively, $\mathrm{D}$ is the bulk partition coefficient of the element between the crystallizing mineral assemblage and the residual melt, $\mathrm{C}^{*}{ }_{\mathrm{L}}$ and
$\mathrm{C}^{*}{ }_{\mathrm{o}}$ represent the content of a hygromagmatophile element in the residual and parental melts, respectively, whose $\mathrm{D}^{*}$ is supposed to equal to zero. Therefore, plotting $\ln \mathrm{C}_{\mathrm{L}}$ against $\ln C^{*}{ }_{L}$ gives a straight line, given $\mathrm{D}$ remains constant during fractional crystallization (Allégre et al. 1977; Christiansen et al. 1983; Fowler and Jensen 1989). Using least-square regression, the slope $(\mathrm{m})$ of the regressed line can be obtained (Fig. 12). Hence, the bulk partition coefficient (D) can be determined for that element (i.e. $m=1-D$ ).

The $\mathrm{D}$ calculation results indicate that $\mathrm{Au}(1.14)$ together with $\mathrm{Sr}$ (1.81), $\mathrm{Ba}$ (2.73), $\mathrm{Hf}$ (1.27), Zr (1.53), La (1.20), Ce (1.12), $\mathrm{Pr}$ (1.31), Eu (3.29), Sc (1.65), Zn (1.21), and $\mathrm{Pb}$ (1.24) were compatible elements during magmatic evolution, suggesting that there is less chance to find Au mineralization in the evolved MPG. Thus, it is better to look for gold within and/or around the early MBG. Based on their D values, $\mathrm{Rb}(0.34)$, Cs (0.80), $\mathrm{Ga}(0.70), \mathrm{Ta}(0.16), \mathrm{Nb}(0.22), \mathrm{Th}(0.56), \mathrm{Y}(0.22), \mathrm{Sm}$ (0.83), Gd (0.6), Yb (0.21), Lu (0.23), Sn (-0.18), W (0.96), Cu $(0.52)$, and $\mathrm{Mo}(0.60)$ behaved as incompatible elements during magmatic evolution.

The technique of Allégre et al. (1977) applied to granitoid systems, such as those in the Mount Pleasant Caldera, may not be interpreted easily. Firstly, the mobility of $U$ in such subvolcanic systems must be considered during late- to post-magmatic hydrothermal alteration processes, although a large proportion of $U$ is hosted in zircon in the MBG and MPG. Incompatibility of Th in a granitoid system is fairly similar to that of $U$, but $T h$ is considered to be immobile during alteration processes (see 
Fowler and Jensen 1989; Jenner 1996). Therefore, Th is a better fractionation index than U. However, using Th as the fractionation index to conduct the same modeling as $U$ described above gave the same tendency of compatibility for all elements listed above. Secondly, the fact that some elements such as Sn, $\mathrm{Sb}, \mathrm{Cu}$, and $\mathrm{Au}$ (see Jenner 1996) would partition into volatile phases during crystallization of the magma, must be also considered in the trace-element simulation. This consideration suggests that a significant fraction of the metals enters volatile phases that reduce their abundance in the degassed magmas. Thus, compatibility of these elements modeled in this empirical fashion (Allégre et al. 1977) may not really reflect their behaviour in the crystal-melt system alone. It is noticeable that the bulk partition coefficient of $\mathrm{Sn}$ is negative $(-0.18)$. This number itself is meaningless, although it is smaller than 1. Thirdly, alteration may elevate or decrease some elements in altered granitoids, which also influences the modeling results. All these effects must be carefully evaluated before a conclusive determination of $\mathrm{D}$ for a metal during evolution of a granitic magma system as described here can be achieved. Then, these data for estimated metal bulk partition coefficients can be applied to assess the mineralization potential.

It is notable that the MPG has very low $\mathrm{K} / \mathrm{Rb}$ ratios (26-56), requiring fluid involvement in formation of the rocks (Shaw 1970; Dostal and Chatterjee 1995, 2000). Conversely, the MBG has high $\mathrm{K} / \mathrm{Rb}$ ratios (172-376), which are typical for magmatic processes. $\mathrm{Nb} / \mathrm{Ta}$ ratios are lower for the MPG (2.7-7.6) compared to the MBG (7.7-28.6), also requiring fluid involvement in the late-stage granites. Intriguingly, the MPG has relatively constant $\mathrm{Zr} / \mathrm{Hf}$ ratios (12.8-17.1), whereas the MBG shows remarkably higher $\mathrm{Zr} / \mathrm{Hf}$ ratios (24.3-48.0), suggesting that $\mathrm{Zr} / \mathrm{Hf}$ ratios in the residual melts were controlled mainly by fractional crystallization of zircon (Linnen and Keppler 2002), or by fluid fractionation (Dostal and Chatterjee 1995, 2000). $\mathrm{The} \mathrm{Th} / \mathrm{U}$ ratios are lower in the MPG (1.1-1.8) and relatively high in the MBG (3.0-7.1), suggesting that $U$ increases more rapidly than Th, consistent with the above estimation of the bulk partition coefficient (D). In other words, $U$ was more incompatible than Th in the magma system. It is known that $\mathrm{Nb}$ and $\mathrm{Ta}$ are strongly affected by the presence of volatiles and can be considered with $\mathrm{Li}, \mathrm{B}, \mathrm{F}, \mathrm{Rb}$, and $\mathrm{Sn}$ as volatile-associated elements (Manning and Hill 1990). In contrast, $\mathrm{Zr}$ and $\mathrm{Hf}$ are considered to be more refractory, in view of the low solubility of their accessory minerals in peraluminous granitic melts (Watson 1979; Watson and Harrison 1983). P, Ti, Sr, and Ba mainly behave as compatible elements in the granitic system.

\section{Estimation of liquidus temperatures}

Liquidus temperatures of granitic rocks can be estimated from accessory mineral saturation temperatures (Watson and Harrison 1983; Harrison and Watson 1984; Montel 1993). These temperatures are capable of reflecting the mechanism of magmatic evolution. In a fractional crystallization sequence, the liquidus temperatures of successive intrusive phases in a granitoid complex decrease from early to late stage crystalliza-

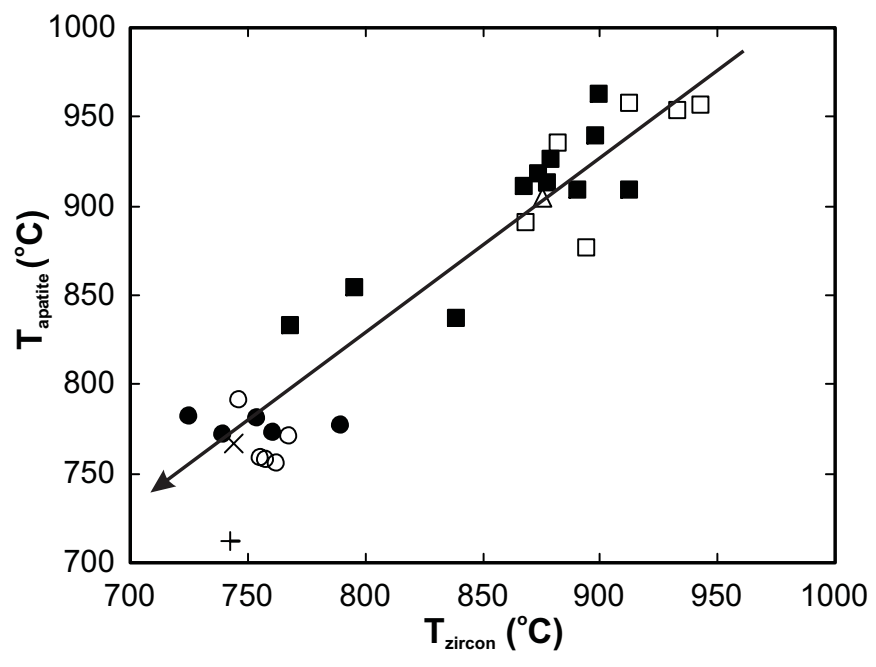

Fig. 15 Diagram of estimated liquidus temperatures $T_{\text {zircon }}$ versus $\mathrm{T}_{\text {apatite }}\left({ }^{\circ} \mathrm{C}\right)$ for granitoid samples from Mount Pleasant Caldera. Symbols are the same as in Figure 4. The arrow points the trend of the temperatures decreasing from the MBG to MPG. Zircon and apatite saturation temperatures $\left({ }^{\circ} \mathrm{C}\right)$ were calculated using Watson and Harrison $(1983)$ and Harrison and Watson (1984) equations, respectively, for the samples listed in Table 1.

tion. On the other hand, the liquidus temperatures of a second stage melt would be higher than a first-stage melt during partial melting, but once formed both evolve by FC related to decreasing temperatures.

Monazite-saturation geothermometry (Montel 1993) yields temperatures as high as $825-950^{\circ} \mathrm{C}$ for the MPG, similar to those of the MBG $\left(834-950^{\circ} \mathrm{C}\right)$. Abnormally high estimated temperatures for the MPG, assuming it formed through fractional crystallization discussed above, are attributed to LREE contents (Table 1 and Fig. 7) similar to those of the MBG. Possibly other unidentified minerals host a significant part of the LREE in the bulk rock. If this portion of LREE were removed from the calculation, then the estimated temperatures would have been lowered in terms of the monazite-saturation geothermometry. However, zircon-saturation geothermometry (Watson and Harrison 1983) produces different results for the MPG $\left(725-789^{\circ} \mathrm{C}\right)$ and MBG $\left(769-933^{\circ} \mathrm{C}\right)$. Apatitesaturation geothermometry (Harrison and Watson 1984) gives results similar to those from zircon-saturation geothermometry for the MPG $\left(712-791^{\circ} \mathrm{C}\right)$ and $\mathrm{MBG}\left(833-963^{\circ} \mathrm{C}\right)$. Decrease of the estimated liquidus temperatures (Fig. 15) from the MBG to MPG is consistent with dropping temperatures in a fractionating magma sequence.

High F concentration (3500-9900 ppm,) together with high $\mathrm{Li}$ (up to $610 \mathrm{ppm}$ ) (see Sinclair and Kooiman 1990) and high alkalis in the water-saturated MPG could remarkably reduce solidus temperatures to as low as $525^{\circ} \mathrm{C}$, as shown by experimental work on F-rich (1.2 wt.\%) topaz-bearing granitic melts (Webster et al. 1987). The large temperature range for crystal- 
lization $\left(950-525^{\circ} \mathrm{C}\right)$ allows protracted extreme fractionation of granitic melts such as the MPG, resulting in unusual enrichment of incompatible elements such as $\mathrm{F}, \mathrm{Li}, \mathrm{Rb}, \mathrm{Cs}, \mathrm{Y}, \mathrm{Nb}, \mathrm{Ta}$, Ga, Sn, and W (Fig. 14).

\section{Discrimination of tectonic setting}

The geochemical features of granitoids may reflect their tectonic environment to some extent (Pearce et al. 1984). Various geochemical discrimination diagrams for tectono-magmatic classification have been proposed to distinguish tectonic settings of magmatic rocks in terms of their chemistry (see Rollinson 1993).

Geochemical discrimination diagrams suggest that the MBG could have formed in volcanic-arc to within-plate tectonic settings, whereas the MPG samples plot entirely in the within-plate field (Figs. 11a and 11b). Figure 11d yields a similar result. As shown above, extensive fractionation plus fluid involvement have strongly enriched high field strength elements, which may shift the MPG samples to the withinplate field (Figs. 11b \& d). Supracrustal contamination also affects the discriminant result (Fig. 11a). Therefore, caution must be exercised when using these diagrams to discriminate tectonic setting. These classification diagrams are suspect (cf. Barker et al. 1992), because they characterize the tectonomagmatic environment under which the granite protolith formed, not necessarily the environment prevailing during granite emplacement (Whalen et al. 1996; Christiansen and Keith 1996). Thus, the trace element signatures of granitoids directly reflect their protolith, melting, and crystallization histories, rather than their tectonic settings. Granitoids from the Mount Pleasant Caldera appear to be crustal A2-type (Fig. 11c), inferred to have been derived from continental crust or underplated crust that has been through a cycle of continent-continent collision or island-arc magmatism (Eby 1990, 1992). Particularly, the MPG is similar in geochemistry to topaz rhyolite (Christiansen et al. 1983, 1984; Christiansen et al. 1986), ongonite, and topaz granite (Kontak 1990; Taylor 1992; Dostal and Chatterjee 1995; Zhu et al. 2001), and also to the Late Devonian Mount Douglas Granite in the eastern part of the Saint George Batholith (Lentz et al. 1988; McLeod et al. 1988; McLeod 1990).

During Late Devonian, the studied region was in the early stages of basin extension (McCutcheon et al. 1990a) followed the Acadian Orogeny, consistent with the discriminant results in terms of Fig. $6 \mathrm{~d}$ and the multicationic diagram of Batchelor and Bowden (1985) (not shown).

\section{Metallogenic implications}

The highly evolved MPG is associated with granophile element mineralization and characterized by high fluorine, lithium (see Sinclair and Kooiman 1990), and alkali concentrations, which enhance the low-T fractionation. The presence of $\mathrm{Li}, \mathrm{B}$, and $\mathrm{F}$ have a significant effect on phase equilibria in the water-saturated granite system $\left(\mathrm{Q}-\mathrm{Ab}-\mathrm{Or}-\mathrm{H}_{2} \mathrm{O}\right)$, reducing liquidus and solidus temperatures and affecting minimum melt compositions (Manning 1981; Webster et al. 1987; Manning and Hill 1990) and decreasing viscosity of the melt (Baker and Vaillancourt 1995), thus promoting the duration of crystallization to low temperatures. On the basis of $\mathrm{F}$ alone, the shift in minimum position toward the $\mathrm{Ab}$ apex in the Q$\mathrm{Ab}$-Or system has been taken as evidence for origin of topaz granite, and other F-rich granitic rocks, through fractional crystallization of biotite granite magma (Manning 1982). F enrichment generally leads to a concomitant enrichment in $\mathrm{Sn}, \mathrm{Nb}$, and $\mathrm{Ta}$, which may possibly be directly complexed with F in the melt (Manning and Hill 1990; Dostal and Chatterjee 1995). A strongly depolymerized F-rich melt is more capable of hosting incompatible elements relative to polymerized volatile-poor granite (Watson 1979; Watson and Harrison 1983; Linnen and Keppler 1997), irrespective of complexing with $F$ (Manning and Hill 1990). Based on the estimated D value of Sn, the late GRIII appears to have high potential for producing Sn mineralization.

Geochemically, the less differentiated MBG is broadly comparable to the granitoids associated with intrusion-related gold systems elsewhere (McCoy et al. 1997; Thompson et al. 1999; Lang et al. 2000; Lang and Baker 2001; Yang et al. 2002a, b). Granitoid intrusions related to gold mineralization are mostly metaluminous, subalkalic (calc-alkalic) to weakly alkalic with intermediate to felsic compositions, forming simple to multiphase complexes (Lang et al. 2000). Locally, peraluminous intrusions are present, but they are ascribed to late-stage fractionation rather than different magmas with distinctive sources (Duncan et al. 1998). According to the summary of Lang et al. (2000), the main-stage, subalkalic (calc-alkalic) intrusions have silica contents between 62 and $72 \mathrm{wt} . \% \mathrm{SiO}_{2}$, and late differentiates reach $78 \mathrm{wt}$. $\% \mathrm{SiO}_{2}$. REE patterns display LREE enrichment relative to $\mathrm{HREE}$, with $\mathrm{La}$ and $\mathrm{Yb}$ concentrations about 100 and 10 times chondrite values, respectively. Eu anomalies are absent to slightly negative. Most intrusions are characterized as reduced I-type granitoids.

Most of the analyzed samples from the MGB and MPG (Table 1) have low Au contents, although the MBG samples appear to contain higher gold (up to $8 \mathrm{ppb}$ ). Compared to continental crust $(2.5 \mathrm{ppb})$ and normal sedimentary rocks, such as greywacke (4.8 ppb) and shale ( $3 \mathrm{ppb}$ ) (see Lentz 2003), Au does not appear to be elevated significantly in the granitoids from the Mount Pleasant Caldera. Regionally, the granitoids in the area also contain relatively low Au compared to continental crust (McLeod 1990; Whalen 1993). Available studies of other intrusion-related Au deposits have shown that the content of $\mathrm{Au}$ in unaltered granitoids is not significantly different from those of barren granitoid rocks (McCoy et al. 1997). These data suggest that background Au abundance in the granitoids is not an essential parameter controlling associated gold mineralization. This observation is in accord with the conclusion by Tilling et al. (1973) that it is impossible to relate gold abundance in igneous rocks and gold mineralization. The geochemical behaviour of gold during the magmatic-hydro- 
thermal evolution of granitoid systems may be a key factor responsible for gold mineralization, which is controlled by the physiochemical conditions and composition of the magmas. Local geological setting (e.g. structure and lithology) and interaction between the intrusions and country rocks affect the magmatic-hydrothermal systems, which ultimately places controls on the emplacement of gold deposits.

Gold is not expected to concentrate in the residual melt during protracted fractionation processes, because it occurs as a compatible metal. Gold is preferentially partitioned into sulphides in equilibrium with a reduced magma, based on empirical observation (McCoy et al. 1997) and experimental studies (Jugo et al. 1999; Kesler et al. 2002). However, early crystallization of magnetite did not appear to severely influence the ability of a calc-alkaline granitic magma to yield a gold-rich ore fluid, according to experimental study of Au partitioning between magnetite and granitic magma (Simon et al. 2003). Consequently, any sulphide saturation in magma systems must greatly deplete Au in the silicate melts. Inverno (1991) reported much higher Au values in his granite samples from the Mount Pleasant Mine, e.g., Au in the GRII ranging from 8 to $85 \mathrm{ppb}$ (average $=28.7 \pm 2.6 \mathrm{ppb}, \mathrm{n}=6$ ), and the GRIII ranging from $<5$ to $27 \mathrm{ppb}$ (average $=13.6 \pm 2.5 \mathrm{ppb}, \mathrm{n}=6$ ) (see his appendix 7). These high Au contents may be ascribed to hydrothermal alteration, although the Au concentrations decrease from the GRII to GRIII. Our greisenized granite samples taken from True Hill also show higher Au contents (up to $100 \mathrm{ppb}$ ), suggesting that late-stage hydrothermal fluids could scavenge gold that is incorporated in early immiscible sulphides in granitoids (Yang and Lentz 2003). At the present stage, it is not easy to evaluate timing of sulphide saturation in felsic magmatic systems, in particular in granite intrusions that were usually influenced by subsolidus processes. These processes may alter the primary sulphides on one hand, and form hydrothermal sulphides on the other. To date, few convincing methods exist for distinguishing primary and hydrothermal sulphides in granitoids, although studies on petrography, chemical, and sulphur isotopic compositions of the sulphides could provide some clues as to their origin. More work needs to be done to better understand the origin of intrusion-related Au ore deposits.

\section{CONCLUSIONS}

Based on the present study, it is suggested that subvolcanic granitoid rocks in the Late Devonian Mount Pleasant Caldera were formed by extreme fractional crystallization of magmas that may have been derived from recycled crustal materials, although the early MBG may have been influenced by local contamination of wall-rocks through selective assimilation. The fractional crystallization is interpreted to have taken place mainly at depth, as indicated by two separate compositional trends for the MBG and MPG. Extensive liquid fractionation may have played an important role in magmatic-hydrothermal evolution, especially facilitating enrichment of high field strength elements, such as $\mathrm{Nb}, \mathrm{Ta}, \mathrm{Y}, \mathrm{HREE}, \mathrm{Sn}$, and W, as well as large ion lithophile elements (e.g. $\mathrm{Li}, \mathrm{Cs}, \mathrm{Rb}$ ) in late-stage melts (i.e. the MPG). High volatile (i.e. F, $\mathrm{Li}$ and $\mathrm{H}_{2} \mathrm{O}$ ) concentrations in the residual melts enhanced the protracted low- $\mathrm{T}$ fractional crystallization. Metal elements behaved distinctively during magmatic evolution. Gold occurred as a compatible element, suggesting that gold and metal mineralization are associated with the early granitoid phases such as the MBG, whereas tin behaved as a very incompatible element so that highly evolved granite phases such as the MPG are more likely to generate tin and related mineralization. Hence, it is suggested that exploration for gold related to granitoid complexes such as those in the Mount Pleasant Caldera should be concentrated around the early phase granitoids. In contrast, the late, highly evolved granite may be a good target for exploration for SnW-Mo-Bi deposits. It is noteworthy that the youngest phase, GRIII, of the MPG seems to have highest potential for forming Sn deposits.

Some questions remain to be answered, in particular the relationship of the granitoids in the Mount Pleasant Caldera to those in the Saint George Batholith and the Pomeroy Intrusive Suite (McLeod 1990). More detailed petrochemical investigations and reliable age determinations (e.g. U-Pb zircon or monazite) are required to characterize the petrogenetic linkages among these granitoids, although they generally have been thought to be related temporally, spatially, and thus genetically (Lentz et al. 1988; Lentz and McAllister 1990; McLeod 1990).

\section{ACKNOWLEDGMENTS}

The authors thank G. Chi (University of Regina), M.J. McLeod, K. G. Thorne, and L.R. Fyffe (NBDNR) for their thoughtful discussions on the geology and intrusion-related gold systems in southwestern New Brunswick. Thanks are also due to Cliff Shaw for constructive discussion about the physical processes operating in a felsic magma chamber. Reviews of the first draft of the manuscript by M.J. McLeod and Cliff Shaw are greatly acknowledged. We are grateful to G.J.A. Kooiman (Geological Consultant; St. George) for discussion of local geology and help with sampling drill core at the Mount Pleasant Mine. R. Young (Manager of the Mount Pleasant Mine) is thanked for allowing us access to the drill core and geological information on site. S.H. McClenaghan and S.E. Watters are thanked for their help with fieldwork. Critical reviews and constructive suggestions by the journal reviewers Drs. Ken Currie and Dan Kontak significantly improved the manuscript. We are grateful to Prof. Sandra M. Barr for editorial handling of the manuscript. This research is funded by the Geological Survey of Canada - Targeted Geoscience Initiative program (GSC-TGI, 010008) and Natural Sciences and Engineering Research Council of Canada grants to D.R. Lentz, with partial support from the New Brunswick Department of Natural Resources and Energy, New Brunswick Innovation Fund, and the University of New Brunswick. 


\section{REFERENCES}

Allégre, C.H., Treuil, M., Minster, J.F., Minster, B., \& Albarede, F. 1977. Systematic use of trace elements in igneous process, Part I: fractional crystallization processes in volcanic suites. Contributions to Mineralogy and Petrology, 60, pp. 57-75.

Anderson, H.E. 1992. A chemical and isotopic study of the age, petrogenesis and magmatic evolution of the Mount Pleasant caldera complex, New Brunswick. Unpublished Ph.D. thesis, Carleton University, Ottawa, Ontario. 203 p.

BaKer, D.R., \& Vaillancourt, J. 1995. The low viscosities of $\mathrm{F}+\mathrm{H}_{2} \mathrm{O}$-bearing granitic melts and implications for melt extraction and transport. Earth and Planetary Science Letters, 132, pp. 199-211.

Barker, F., Farmer, G.L., Ayuso, R.A., Plafker, G., \& Lull, J.S. 1992. The 50 Ma granodiorite of the eastern Gulf of Alaska: melting in an accretionary prism in the forarc. Journal of Geophysical Research, 97, pp. 6757-6778.

BARR, S.M., \& White, C.E. 1999. Field relations, petrology, and structure of Neoproterozoic rocks in the Caledonian Highlands, southern New Brunswick, Canada. Geological Survey of Canada Bulletin 530, $101 \mathrm{p}$.

Barr, S.M., White, C.E., \& Miller, B.V. 2002. The Kingston terrane, south New Brunswick, Canada: evidence for an Early Silurian volcanic arc. Geological Society of America Bulletin, 114, pp. 964-982.

BAtchelor, R.A., \& Bowden, P. 1985. Petrogenetic interpretation of granitoid rock series using multicationic parameters, Chemical Geology, 48, pp.43-55.

Brophy, J.G. 1991. Composition gaps, critical crystallinity, and fractional crystallization in orogenic (calc-alkaline) magmatic systems. Contributions to Mineralogy and Petrology, 109, pp. 173-182.

CHI, G. 2002. Fluid compositions and temperature-pressure conditions of intrusion-related gold systems in southwestern New Brunswick a fluid inclusion study. Geological Survey of Canada, Current Research 2002-E13, 11 p.

Christiansen, E.H., \& Keith, J.D. 1996. Trace-element systematics in silicic magmas: a metallogenic perspective. In Trace Element Geochemistry of Volcanic Rocks: Applications for Massive Sulfide Exploration. Edited by D.A. Wyman. Geological Association of Canada, Short Course Notes 12, pp. 115-151.

Christiansen, E.H., Burt, D.M., Sheridan, M.F., \& WiLson, R.T. 1983. The petrogenesis of topaz rhyolites from the western United States. Contributions to Mineralogy and Petrology, 83, pp. 16-30.

Christiansen, E.H., Bikun, J.V., Sheridan, M.F., \& Burt, D.M. 1984. Geochemical evolution of topaz rhyolites from the Thomas Range and Spor Mountain, Utah. American Mineralogist, 69, pp. 223-236.

Christiansen, E.H., Sheridan, M.F., \& Burt, D.M. 1986. The geology and geochemistry of Cenozoic topaz rhyolites from the western United States. Geological Society of America, Special Paper 205, pp. 1-82.
Clarke, D.B. 1992. Granitoid rocks. London, Chapman \& Hall. 283 p.

Collins, W.J., Beams, S.D., White, A.J.R., \& Chappell, B.W. 1982. Nature, and origin of A-type granites with particular reference to southeastern Australia. Contributions to Mineralogy and Petrology, 80, pp. 189-200.

Davis, W.J., \& Williams-Jones, A.E. 1985. A fluid inclusion study of the porphyry-greisen, tungsten-molybdenum deposit at Mount Pleasant, New Brunswick, Canada. Mineralium Deposita, 20, pp. 94-101.

Dostal, J., \& Chatterjee, A.K. 1995. Origin of topaz-bearing and related peraluminous granites of late Devonian Davis Lake pluton, Nova Scotia, Canada. Chemical Geology, 123, pp. 67-88.

Dostal, J., \& Chatterjee, A.K. 2000. Contrasting behaviour of $\mathrm{Nb} / \mathrm{Ta}$ and $\mathrm{Zr} / \mathrm{Hf}$ ratios in a peraluminous granitic pluton (Nova Scotia, Canada). Chemical Geology, 163, pp. 207-218.

Duncan, R.A., Russell, J.K., Hastings, N.L., \& Anderson, R.G. 1998. Relationships between chemical composition, physical properties and geology of the mineralized Emerald Lake Pluton, Yukon. Current Research, Geological Survey of Canada, Paper 98-9A. pp. 1-11.

Eву, G.N. 1990. The A-type granitoids: a review of their occurrence and chemical characteristics and speculations on their petrogenesis. Lithos, 26, pp. 115-134.

Еву, G.N. 1992. Chemical subdivision of the A-type granitoids: petrogenetic and tectonic implications. Geology, 20, pp. 640-644.

Fan, H.R., Zhai, M.G., XIE, Y.H., \& YANG, J.H. 2003. Oreforming fluids associated with granite-hosted gold mineralization at the Sanshandao deposit, Jiaodong gold province, China. Mineralium Deposita, 38, pp. 739-750.

FenN, P.M. 1986. On the origin of graphic granite. American Mineralogist, 71, pp. 325-330.

Fowler, A.D., \& JENSEN, L.S. 1989. Quantitative trace-element modelling of the crystallization history of the Kinojevis and Black River groups, Abitibi Greenstone Belt, Ontario. Canadian Journal of Earth Sciences, 26, pp. 1356-1367.

Frost, B.R., Barnes, C.G., Collins, W.J., Arculus, R.J., Ellis, D.J., \& Frost, C.D. 2001. A geochemical classification for granitic rocks. Journal of Petrology, 42, pp. 20332048.

FyFFE, L.R., \& FrICKER, A. 1987. Tectonostratigraphic terrane analysis of New Brunswick. Maritime Sediments and Atlantic Geology, 23, pp. 113-122.

Fyffe, L.R., Pickerill, R.K., \& Stringer, P. 1999. Stratigraphy, sedimentology and structure of the Oak Bay and Waweig formations, Mascarene Basin: implications for the paleotectonic evolution of the southwestern New Brunswick. Atlantic Geology, 35, pp. 59-84.

Gorton, M.P., \& SCHANDL, E.S. 2000. From continents to island arcs: a geochemical index of tectonic setting for arc-related and within-plate felsic to intermediate volcanic rocks. Canadian Mineralogist, 38, pp. 1065-1073. 
Gottini, V. 1968. The $\mathrm{TiO}_{2}$ frequency in volcanic rocks. Geologische Rundschau, 57, pp. 930-935.

Govindaraju, K. 1994. Compilation of working values and sample description for 383 Geostandards. Geostandards Newsletter, 18, Special Issue, pp. 1-158.

Harrison, T.M., \& Watson, E.B. 1984. The behaviour of apatite during crustal anatexis: equilibrium and kinetic considerations. Geochimica et Cosmochimica Acta, 48, p. 1467-1477.

INVERNO, C.M.C. 1991. Endogranitic tin mineralization in Mount Pleasant intrusive complex, New Brunswick, Canada. Unpublished PhD thesis, Colorado School of Mines, Golden, Colorado, USA. 302 p.

Jenner, G.A. 1996. Trace element geochemistry of igneous rocks: geochemical nomenclature and analytical geochemistry. In Trace Element Geochemistry of Volcanic Rocks: Applications for Massive Sulfide Exploration. Edited by D.A. Wyman. Geological Association of Canada, Short Course Notes 12, pp. 51-77.

Johnson, S. 2003. Conceptual models and potential gold environments in the Carboniferous Rocks of New Brunswick. $105^{\text {th }}$ CIM Annual Meeting, Abstract with Programs, p. 198.

Jugo, P.J., Candela, P.A., \& Piccoli, P.M. 1999. Magmatic sulfides and $\mathrm{Au}: \mathrm{Cu}$ ratios in porphyry deposits: an experimental study of copper and gold partitioning at $850^{\circ} \mathrm{C}, 100$ $\mathrm{MPa}$ in a haplogranitic melt-pyrrhotite-intermediate solid solution-gold metal assemblage, at gas saturation. Lithos, 46, pp. 573-589.

Kesler, S.E., Chryssoulis, S.L., \& Simon, G. 2002. Gold in porphyry copper deposits: its abundance and fate. Ore Geology Reviews, 21, pp. 103-124.

KIRKHAM, R.V., \& SinClaIR, W.D. 1988. Comb quartz layers in felsic intrusions and their relationship to porphyry deposits. In Recent Advances in the Geology of Granite-Related Mineral Deposits. Edited by R.P. Taylor and D.F. Strong. Canadian Institute of Mining and Metallurgy Special Volume 39, pp. 50-71.

KonTAK, D.J. 1990. The East Kemptville topaz-muscovite leucogranite, Nova Scotia I. Geological setting and whole-rock geochemistry. Canadian Mineralogist, 28, pp. 787-825.

Kontak, D.J., \& Clark, A.H. 2002. Genesis of the giant, Bonanza San Rafael lode tin deposit, Peru: Origin and Significance of Pervasive alteration. Economic Geology, 97, pp. 1741-1777.

Kooiman, G.J.A., McLeod, M.J., \& Sinclair, W.D. 1986. Porphyry tungsten-molybdenum ore bodies, polymetallic veins and replacement bodies, and tin-bearing greisen zones in the Fire Tower Zone, Mount Pleasant, New Brunswick. Economic Geology, 81, pp. 1356-1373.

LANG, J.R., \& BAKER, T. 2001. Intrusion-related gold systems: the present level of understanding. Mineralium Deposita, 36, pp. 477-489.

Lang, J.R., Baker, T., Hart, C.J., \& Mortensen, J.K. 2000. An exploration model for intrusion-related gold systems.
Society of Economic Geologists Newsletter, No. 40, pp. 1-15.

Le Maitre, R.W., Bateman P., Dudek A., Keller J., Lameyre J., Le Bas M.J., Sabine P.A., Schmid R., Sorensen H., Streckeisen A., Wooley A.R., \& ZanetTIN B. 1989. A Classification of Igneous Rocks and Glossary of Terms, Blackwell, Oxford, 193 p.

LENTZ, D.R. 1994. Multiple episodes of brecciation and mineralization associated with an epizonal granite porphyry, True Hill, southwestern New Brunswick. Exploration and Mining Geology, 3, pp. 9-16.

LENTZ, D.R. 2003. Geochemistry of sediments and sedimentary rocks: historical to research perspectives. In Inorganic geochemistry of sediments and sedimentary rocks: evolutionary considerations to mineral deposit-forming environments. Edited by D.R. Lentz. Geological Association of Canada, GeoText 4, pp. 1-6

Lentz, D.R., \& Fowler, A.D. 1992. A dynamic model for graphic quartz-feldspar intergrowths in granitic pegmatites in the southwestern Grenville Province. Canadian Mineralogist, 30, pp. 571-585.

Lentz, D.R., \& Gregoire, C. 1995. Petrology and mass-balance constraints on major-, trace-, and rare-earth-element mobility in porphyry-greisen alteration associated with the epizonal True Hill granite, southwestern New Brunswick, Canada. Journal of Geochemical Exploration, 52, pp. 303-331.

LenTZ, D.R., \& McAllister, A.L. 1990. The petrogenesis of tin- and sulfide-lode mineralization at True Hill, southwestern New Brunswick. Atlantic Geology, 26, pp. 139-155.

Lentz, D.R., Lutes, G., \& Hartree, R. 1988. Bi-Sn-Mo-W greisen mineralization associated with the True Hill granite, southwestern New Brunswick. Maritime Sediments and Atlantic Geology, 24, pp. 321-338.

LentZ, D.R., Thorne, K.G., \& YAng, X.M. 2002. Preliminary analysis of the controls on the various episodes of gold mineralization at the Lake George antimony deposit, New Brunswick. In Current Research 2002. Edited by B.M.W. Carroll. New Brunswick Department of Natural Resources and Energy Division, Mineral Resource Report 02-1, pp. $55-79$.

Linnen, R.L., \& Keppler, H. 1997. Columbite solubility in granitic melts: consequences for the enrichment and fractionation of $\mathrm{Nb}$ and $\mathrm{Ta}$ in the Earth's crust. Contributions to Mineralogy and Petrology, 128, pp. 213-227.

Linnen, R.L., \& KePpleR, H. 2002. Melt composition control of $\mathrm{Zr} / \mathrm{Hf}$ fractionation in magmatic processes. Geochimica et Cosmochimica Acta, 66, pp. 3293-3301.

LONGERICH, H.P. 1995. Analysis of pressed powder pellets of geological samples using wavelength-dispersive Xray fluorescence spectrometry. X-ray Spectrometry, 24, pp. 123-136.

ManNing, D.A.C. 1981. The effect of fluorine on liquidus phase relationships in the system Qz-Ab-Or with excess water at $1 \mathrm{~kb}$. Contributions to Mineralogy and Petrology, 76, pp. 206-215. 
ManNing, D.A.C. 1982. An experimental study of the effects of fluorine on the crystallization of granitic melts. In Metallization associated with acid magmatism. Edited by A.M. Evans. Chichester, UK, John Wiley, pp. 191-203.

ManNing, D.A.C. 1988. Late-stage granitic rocks and mineralization in southwest England and southeast Asia. In Recent advances in the Geology of granite-related mineral deposits. Edited by R.P. Taylor and D.F. Strong. The Canadian Institute of Mining and Metallurgy, Special Volume 39 , pp. 80-85.

ManNing, D.A.C., \& Hill, P.I. 1990. The petrogenetic and metallogenetic significance of topaz granite from the southwest England orefield. In Ore-bearing granite systems; Petrogenesis and mineralizing processes. Edited by H.J. Stein and J.L. Hannah. Geological Society of America, Special Paper 246, pp. 51-69.

Marsh, B.D. 1996. Solidification fronts and magmatic evolution. Mineralogical Magazine, 60, pp. 5-40.

McCoy, D., Newberry, R.J., Layer, P., DiMarchi, J.J., Bakke, A, Masterman, J.S., \& Minehane, D.L. 1997. Plutonic-related gold deposits of Interior Alaska. Economic Geology Monograph 9, pp. 191-241.

McCutcheon, S.R. 1990a. The Late Devonian Mount Pleasant caldera complex: stratigraphy, mineralogy, geochemistry and geologic setting of a Sn-W deposit in southwestern New Brunswick. unpublished Ph.D. thesis, Dalhousie University, Halifax, NS. 609 p.

McCutcheon, S.R. 1990b. The Mount Pleasant Caldera: geological setting of associated tungsten-molybdenum and tin deposits. In Mineral deposits of New Brunswick and Nova Scotia [field trip 2], $8^{\text {th }}$ IAGOD Symposium field trip guidebook. Edited by D.R. Boyle. Geological Survey of Canada, Open File 2157, pp. 73-77.

McCutcheon, S.R., ANderson, H.E., \& Robinson, P.T. 1997. Stratigraphy and eruptive history of the Late Devonian Mount Pleasant Caldera Complex, Canadian Appalachians. Geological Magazine, 134, pp. 17-36.

McCutcheon, S.R., Sinclair, W.D., \& Kooiman, G.J.A. 2001. The Late Devonian Mount Pleasant Caldera: geological setting of a W-M0-Sn-In deposit in southwestern New Brunswick. In Guidebook to field trips in New Brunswick and western Maine. Edited by R.K. Pickerill and D.R. Lentz. 93rd Annual Meeting New England Intercollegiate Geological Conference, B5-B14.

McLeod, M.J. 1990. Geology, geochemistry, and related mineral deposits of the Saint George Batholith; Charlotte, Queens, and Kings Counties, New Brunswick. New Brunswick Department of Natural Resources and Energy, Mineral Resources, Mineral Resource Report 5, 169 p.

MCLEOD, M.J. 2002. An emerging gold district in southern New Brunswick: tectonic setting, models and future prospects. Prospectors and Developers Association of Canada, International Convention, Trade Show, and Investors Exchange 2002 (Toronto), Technical Program and Core Shack Abstract, p. 15.

McLeod, M.J., \& FyfFe, L.R. 2002. Geology and gold occurrences, Clarence Stream area, southwestern New Bruns- wick. New Brunswick Department of Natural Resources and Energy; Minerals, Policy and Planning Division, Plate 38A.

McLeod, M.J., \& McCutcheon, S.R. 2000. Gold environments in New Brunswick. New Brunswick Department of Natural Resources and Energy, Minerals and Energy Division, Map Plate 2000-28.

McLeod, M.J., TAYLOR, R.P., \& Lux, D.R. 1988. Geology, Ar/ Ar geochronology and Sn-W-Mo-bearing sheeted veins of the Mount Douglas Granite, southwestern New Brunswick. Canadian Institute of Mining and Metallurgical Bulletin, 81, pp. 70-77.

MonTEL, J.M. 1993. A model for monazite/melt equilibrium and application to the generation of granitic magmas. Chemical Geology, 110, pp. 127-146.

PеAсоск, M.A. 1931. Classification of igneous rock series. Journal of Geology, 39, pp. 54-67.

Pearce, J.A, Harris, N.B.W., \& Tindle, A.G. 1984. Trace element discrimination diagrams for the tectonic interpretation of granitic rocks. Journal of Petrology, 25, pp. 956-983.

QIU, J.X. 1986. Magmatic petrology. Beijing, Geological Publishing House, $366 \mathrm{p}$.

Rittmann, A. 1957. On the serial character of igneous rocks. Egyptian Journal of Geology, 1, pp. 23-48.

RitTmanN, A. 1973. Stable mineral assemblages of igneous rocks. Berlin, Springer- Verlag. 262 p.

RoLLINSON, H.R. 1993. Using geochemical data: evaluation, presentation, interpretation, London, Longman Scientific $\&$ Technical. $552 \mathrm{p}$.

SAMSON, I.M. 1990. Fluid evolution and mineralization in a subvolcanic granite stock: the Mount Pleasant W-Mo-Sn deposits, New Brunswick, Canada. Economic Geology, 85, pp. 145-163.

SAUNDERs, A.D., \& TARNEY, J. 1984. Geochemical characteristics of basaltic volcanism within back-arc basins. In Marginal basin geology. Edited by B.P. Kokelaar and M.F. Howells. Special Publication of the Geological Society of London, 16, pp. 59-76.

SHAw, D.M. 1970. Trace element fractionation during anatexis. Geochimica et Cosmochimica Acta, 37, pp. 237-243.

Simon, A.C., Pettke, T., Candela, P.A., Piccoli, P.M., \& HeInrich, C.A. 2003. Experimental determination of Au solubility in rhyolite melt and magnetite: Constraints on magmatic Au budgets. American Mineralogist, 88, pp. 1644-1651.

Sinclair, W.D., \& Kooiman, G.J.A. 1990. The Mount Pleasant tungsten-molybdenum and tin deposits. In Mineral deposits of New Brunswick and Nova Scotia [field trip 2], $8^{\text {th }}$ IAGOD Symposium field trip guidebook. Edited by D.R. Boyle. Geological Survey of Canada, Open File 2157, pp. 78-87.

Sinclair, W.D., Koolman, G.J.A., \& Martin, D.A. 1988. Geological setting of granites and related tin deposits in the North Zone, Mount Pleasant, New Brunswick. Geological Survey of Canada, Current Research, Part B, Paper 88-1B, pp. 201-208. 
Sun, S.S. 1980. Lead isotopic study of young volcanic rocks from mid-ocean ridges, ocean islands and island arcs. Philosophical Transactions of the Royal Society, A297, pp. 409-445.

Sun, S.S. 1982. Chemical composition and origin of the Earth's primitive mantle. Geochimica et Cosmochimica Acta, 46, pp. 179-192.

TAYLOR, R.P. 1992. Petrological and geochemical characteristics of the Pleasant Ridge zinnwaldite topaz granite, southern New Brunswick, and comparisons with other topaz-bearing felsic rocks. Canadian Mineralogist, 30, pp. 895-921.

Taylor, S.R., \& McLennan, S.M. 1985. The Continental Crust: Its Composition and Evolution, Blackwell, Oxford. $277 \mathrm{p}$.

TAYLOR, R.P., Sinclair, W.D., \& LUTES, G. 1985. Geochemical and isotopic characterization of granites related to W-Sn-Mo mineralization in the Mount Pleasant area, New Brunswick. In Granite-related mineral deposits: Geology, petrogenesis and tectonic setting. Edited by R.P. Taylor and D.F. Strong. CIM extended abstracts, pp. 265-273.

Thompson, J.F.H., Sillitoe, R.H., BAKER, T., LANG, G J.R., \& Mortensen, J.K. 1999. Intrusion-related gold deposits associated with tungsten tin provinces. Mineralium Deposita, 34, pp. 323-334.

Thorne, K.G., \& LENTZ, D.R. 2001. Geological setting of the Clarence Stream gold deposit, southwestern New Brunswick. In Guidebook of field trips in New Brunswick and Eastern Maine, New England Intercollegiate Geological Conference 2001. Edited by R.K. Pickerill and D.R. Lentz. $93^{\text {rd }}$ Annual Meeting, pp. C5-1-C5-16.

Thorne, K.G., \& LenTZ, D.R. 2002. Geochemistry and petrogenesis of the East Branch Brook metagabbroic dykes in the Sawyer Brook fault zone, Clarence Stream gold prospect, southwestern New Brunswick. Atlantic Geology, 37, pp. 175-190.

ThORne, K.G., \& Lentz, D.R. 2003. Geology of the Main Zone of the Clarence Stream gold deposit, southwestern New Brunswick. In Gold deposits associated with felsic intrusions in southwestern New Brunswick - field guidebook New Brunswick Department of Natural Resources and Energy. Edited by K.G. Thorne and M.J. McLeod. Minerals, Policy and Planning Division, Open File 2003-4, pp. 13-29.

Thorne, K.G., Lentz, D.R., Hall, D.C., \& Yang, X.M. 2002. Petrology, geochemistry, and geochronology of the granitic pegmatite and aplite dykes associated with the Clarence Stream gold deposit, southwestern New Brunswick. Geological Survey of Canada, Current Research 2002-E12, $13 \mathrm{p}$.

Tilling, R.I., GotTFried, D., \& Rowe, J.J. 1973. Gold abundance in igneous rocks: bearing on gold mineralization. Economic Geology, 68, pp. 168-186.

Tucker, R.D., Bradley, D.C., ver Straeten, C.A., Harris, A.C., Ebert, J.R., \& McCutcheon, S.R. 1998. New U-Pb zircon ages and the duration and division of Devonian time. Earth and Planetary Science Letters, 158, pp. 175-186.
Watson, E.B. 1979. Zircon saturation in felsic liquids: experimental results and application to trace element geochemistry. Contributions to Mineralogy and Petrology, 70, pp. 407-419.

Watson, E.B., \& Harrison, T.M. 1983. Zircon saturation revisited: temperature and composition effects in a variety of crustal magma types. Earth and Planetary Science Letters, 64, pp. 295-304.

WEAVER, B., \& TARNEY, J. 1984. Empirical approach to estimating the composition of the continental crust. Nature, 310, pp. 575-577.

Webster, J.D., Holloway, J.R., \& Hervig, R.L. 1987. Phase equilibria of a Be, $U$ and $F$ enriched vitrophyre from Spor Mountain, Utah. Geochimica et Cosmochimica Acta, 51, pp. 389-402.

WhaLen, J.B. 1993. Geology, petrography, and geochemistry of Appalachian granites in New Brunswick and Gaspésie, Quebec. Geological Survey of Canada Bulletin 436, 124 p.

Whalen, J.B., Currie, K.L., \& Chappell, B. W. 1987. A-type granites: geochemical characteristics, discrimination and petrogenesis. Contributions to Mineralogy and Petrology, 95, pp. 407-419.

Whalen, J.B., Fyffe, L.R., Longstaffe, F.J., \& Jenner, G.A. 1996. The position and nature of the Gander-Avalon boundary, southern New Brunswick, based on geochemical and isotopic data from granitoid rocks. Canadian Journal of Earth Sciences, 33, pp. 129-139.

White, A.J.R., \& ChAppell, B.W. 1983. Granitoid types and their distribution in the Lachlan Fold Belt, southeastern Australia. Geological Society of America, Memoir no.159, pp. 21-33.

YANG, X.M., \& LENTZ, D.R. 2003. Geochemical behaviour of metals during magmatic-hydrothermal evolution of intrusion-related gold systems, southwestern New Brunswick. The 28th Annual Review of Activities, New Brunswick Department of Natural Resources and Energy, Minerals, Policy and Planning Division, Fredericton, Abstracts with program, p. 65-66.

YANG, X.M., Lentz, D.R., \& CHI, G. 2002a. Petrochemistry of Lake George granodiorite stock and related Au mineralization, York County, New Brunswick. Geological Survey of Canada, Current Research 2002-D7, 10 p.

Yang, X.M., Lentz, D.R., Hall, D.C., \& Chi, G. 2002b. Petrology of the Lake George granodiorite stock, New Brunswick: implications for crystallization conditions, volatile exsolution, and W-Mo-Au-Sb mineralization. Geological Survey of Canada, Current Research 2002-E14, 12 p.

Zhu, J.C., Li, R.K., Li, F.C., Xiong, X.L., Zhou F.Y., \& HuANG, X.L. 2001. Topaz-albite granites and rare-metal mineralization in the Limu District, Guangxi, Province, southeast China. Mineralium Deposita, 36, pp. 393-405.

Editorial responsibility: Sandra M. Barr 
